\title{
Synthesis and characterization of Mn/Co/Ti LDH and its utilization as a photocatalyst in visible light assisted degradation of aqueous Rhodamine $\mathrm{B} \dagger$
}

\author{
Priyadarshi Roy Chowdhury and Krishna G. Bhattacharyya* \\ Department of Chemistry, Gauhati University, Guwahati 781014, Assam, India.
}

\begin{abstract}
Luminescent $\mathrm{Mn} / \mathrm{Co} / \mathrm{Ti} \mathrm{LDH}$, synthesized by a single step hydrothermal route, has been found to be optically responsive for utilization as a highly efficient photocatalyst in destruction of the cationic dye Rhodamine B, in aqueous solution under visible light irradiation. The material has been found to be better than the commercial catalysts like $\mathrm{MnO}, \mathrm{CoO}, \mathrm{TiO}_{2}$ and Degussa P25. Multiple techniques like XRD, XPS, FT-IR, EIS, TG, UV-visible DRS, PL, TRES, $\mathrm{N}_{2}$-sorptiondesorption, dynamic light scattering, TEM-EDS and AFM analyses were used to characterize the $\mathrm{LDH}$. The results indicated $\mathrm{Mn} / \mathrm{Co} / \mathrm{Ti} \mathrm{LDH}$ to have a multilayered crystalline structure with hexagonal morphology that carries metal ions in mixed valences, oxygen vacancies, defect states, thermal stability, narrow band gap, high surface area, and electrostatic surface charge variation with $\mathrm{pH}$. The photocatalytic activity of the LDH could be co-related with the structural aspects such as oxidation states, narrow band gap, high surface area and existence of defects. The active species responsible for photocatalysis have been evaluated with EPR, terephthalic acid fluorescence probe and indirect radical-hole trapping experiments. The photodegradation mechanism involves electron and hole hopping across the material and also photosensitization of the dye. Ex situ ${ }^{1} \mathrm{H}-\mathrm{NMR}$ and GC-MS analyses of the colorless end products of Rhodamine B destruction provide further insight into the reaction mechanism. The complete mineralization of the decolorized end product of degradation was evaluated with TOC analysis. The results indicate the potential for using multi metal incorporated LDH in destroying dyes and their degradation products in industrial wastewater.
\end{abstract}

\section{Introduction}

Photocatalysis occurs through the process of generation of electrons and holes in the appropriate materials. ${ }^{1}$ The widespread importance of heterogeneous photocatalysis has been thought to be due to the consequences of the Fujishima-Honda effect (1972). ${ }^{2}$ Photocatalysis has been explored as a solution to deal with problems of large scale environmental pollution. ${ }^{3}$ It has been shown that visible light induced photocatalysis could be cost effective in treating with effluents containing a wide variety of organics and dyes. ${ }^{4,5}$ Dyes are the copious sources of coloured organics extensively used in textile industries, and

\footnotetext{
$\dagger$ Electronic supplementary information (ESI) available: X-ray diffraction data, characterizations and photocatalysis results associated with this work. See DOI: $10.1039 / \mathrm{c} 6 \mathrm{ra} 24288 \mathrm{j}$

kgbhattacharyya@gmail.com; Fax: +91 361 2570599; Tel: +91 9864031987.
} 
discharged in comparatively high concentration in the effluents, making their removal ineffective in the conventional train of unit processes like filtration, coagulation, biological oxidation and adsorption on activated carbon, etc. Hence, it has become imperative to develop eco-friendly, simple and economical techniques for the removal of the hazardous dyes from water. ${ }^{6,7}$ One class of materials that have received wide attention in this regard is the layered double hydroxides (LDHs) having the general formula, $\left[\mathrm{M}^{2+}{ }_{1-\mathrm{xM}^{3+}}\right.$ ${ }_{\mathrm{x}}(\mathrm{OH})_{2}\left(\mathrm{~A}^{\mathrm{n}-}\right)_{\mathrm{x} / \mathrm{n}} \mathrm{yH}_{2} \mathrm{O}$, where $\mathrm{M}^{2+}$ and $\mathrm{M}^{3+}$ are divalent and trivalent cations, and $\mathrm{A}^{\mathrm{n}-}$ is the charge balancing interlayer anion. As a result of relatively weak interlayer bonding, $\mathrm{LDH}$ exhibits expanding properties with variability in the composition and finds application in highly efficient photochemical reactions. Due to high surface area, narrow band gap, presence of defect sites along with n-type semiconductor characteristics and high recyclability, the $\mathrm{LDH}$ is expected to play a major role in cost-effective photocatalytic degradation of carcinogenic and neurotoxic dyes from textile industries. $5,7,8$

In this work, the synthesis and characterization of a novel $2: 1: 1 \mathrm{Mn} / \mathrm{Co} / \mathrm{Ti} \mathrm{LDH}$ are reported with application in visible light assisted photodegradation of aqueous Rhodamine B. The mechanism of LDH synthesis is discussed from the viewpoint of nucleation and self assembly, induced by Ostwald ripening. ${ }^{8}$ Characterizations of the LDH shows an effective separation of $\mathrm{e}^{-}-\mathrm{h}^{+}$pair, narrow band gap, high surface area with defect states, and all these properties have been suggested to contribute to its remarkable performance as a photocatalyst. On the basis of the results, valuable insights into the mechanism of photocatalysis of the dye have been suggested with electron-hole hopping conduction model as the basis. The results from ex situ ${ }^{1} \mathrm{H}-\mathrm{NMR}$ and GC-MS analyses of the colorless degradation products of the dye have been used to elucidate the reaction mechanism of Rhodamine B degradation. The work thus exhibits that $\mathrm{Mn} / \mathrm{Co} / \mathrm{Ti} \mathrm{LDH}$ could be an important addition to cost-effective photocatalysts for large scale environmental waste water treatment.

\section{Experimental section}

\subsection{Materials}

All the chemicals used were analytical grade reagents. $\mathrm{TiO}_{2}$ (particle diameter $30 \mathrm{~nm}$, surface area $60 \mathrm{~m}^{2} \mathrm{~g}^{-1}$, band gap $3.17 \mathrm{eV}$; product name Degussa P25) having high levels of photocatalytic activity and available from Degussa Corp. was used as a reference photocatalyst. $\mathrm{MnO}_{2}$ (surface area $90 \mathrm{~m}^{2} \mathrm{~g}^{-1}$, band gap $2.5 \mathrm{eV}$ ) and $\mathrm{CoO}$ (surface area 85 $\mathrm{m}^{2} \mathrm{~g}^{-1}$; band gap $\sim 2.68 \mathrm{eV}$ ), associated with many redox systems, were purchased from Sigma Aldrich, Germany and were also used as reference photocatalysts. $\mathrm{TiO}_{2}$ (anatase phase; surface area $100 \mathrm{~m}^{2} \mathrm{~g}^{-1}$, band gap $\sim 3.26 \mathrm{eV}$ ) being primarily known for its high charge separation and improvement of the photocatalytic performance was purchased from Merck Chemicals Co. USA and was used without further purification as reference catalyst. Decarbonated water was used throughout the experiments.

\subsection{Synthesis of $\mathrm{Mn} / \mathrm{Co} / \mathrm{Ti} \mathrm{LDH}$}

$2: 1: 1 \mathrm{Mn} / \mathrm{Co} / \mathrm{Ti} \mathrm{LDH}$ was synthesized by a single step hydrothermal route taking $10.98 \mathrm{~g}$ $\mathrm{Mn}\left(\mathrm{NO}_{3}\right)_{2} 4 \mathrm{H}_{2} \mathrm{O}, 5.89 \mathrm{~g} \mathrm{Co}\left(\mathrm{NO}_{3}\right)_{2} 6 \mathrm{H}_{2} \mathrm{O}, 1.1 \mathrm{ml} \mathrm{TiCl}_{4}$ and $3.0 \mathrm{~g}$ urea in $100 \mathrm{ml}$ 
decarbonated water, followed by vigorous stirring and subsequent hydrothermal ageing ${ }^{5}$ of the mixture in a Teflon lined autoclave at $140{ }^{\circ} \mathrm{C}$ for $36 \mathrm{~h}$. The crystalline product is extracted, washed with decarbonated water and dried in air at room temperature. The LDH synthesis is schematically illustrated in Fig. 1.

\subsection{Characterizations}

The powder X-ray diffraction (XRD) pattern of the $\mathrm{Mn} / \mathrm{Co} / \mathrm{Ti} \mathrm{LDH}$ was obtained using a PANalytical X'Pert PRO diffractometer using monochromated $\mathrm{CuK}_{\mathrm{a}} \mathrm{X}$-ray radiation $(\lambda=$ $1.5418 \AA$ ) at $25 \mathrm{~mA}$ at $35 \mathrm{kV}$ with a scanning rate of $4^{\circ} \mathrm{min}^{-1}$ and $2 \mathrm{q}$ angle of 5 to $80^{\circ}$. Xray photoelectron spectroscopy (XPS) was done with a PHI Quantum 5900 ESCA instrument in order to identify the chemical states and understand the nature of bonding taking place between the different groups present on the LDH surface. The XPS sample was irradiated with $\mathrm{Al} \mathrm{K}_{\mathrm{a}} \mathrm{X}$-rays under ultrahigh vacuum conditions. FT-IR analysis was performed with a Shimadzu FT-IR 3000 spectrometer, the palette having LDH : KBr in the ratio of $1: 100$. The electrochemical impedance spectroscopy (EIS) analysis was carried out with a $\mathrm{CHI} 760 \mathrm{E}$ electrochemical workstation with a three-electrode configuration, (a) $\mathrm{Pt}$ wire (counter electrode) (b) saturated calomel electrode (SCE; reference electrode) and (c) working electrode (LDH deposited on ITO glass). A $0.5 \mathrm{M} \mathrm{Na}_{2} \mathrm{SO}_{4}$ solution was used as the electrolyte during the impedance measurements. Thermogravimetric analysis (TG) from 32 to $750{ }^{\circ} \mathrm{C}$ was done in a Mettler Toledo Thermal Analyser in an inert atmosphere with a heating rate of $10{ }^{\circ} \mathrm{C} \mathrm{min}-1$. The UV-visible diffuse reflectance spectroscopy of the LDH was investigated on a Hitachi U4100 spectrometer, equipped with an integrating sphere attachment and using $\mathrm{BaSO}_{4}$ background. Photoluminescence (PL) analysis at different excitation wavelengths was investigated using a Hitachi F-2500 FL spectrophotometer with a Xe lamp as the excitation source. Time-resolved photoluminescence (TRPL) measurements were done at room temperature using a PicoQuant Fluo Time 300 lifetime spectrophotometer. The specific surface area and pore size distribution of the LDH was investigated by low temperature multipoint $\mathrm{N}_{2}$-sorption-desorption measurements following Brunauer-Emmett-Teller (BET) method using a Micromeritics Tristar 3000 V6.08 analyzer. Prior to the analysis, the $\mathrm{LDH}$ was degassed at $100{ }^{\circ} \mathrm{C}$ under vacuum for $3 \mathrm{~h}$ to remove the moisture content. The variation of electrostatic surface charge of the $\mathrm{LDH}$ with $\mathrm{pH}$ was measured using a Malvern Zetasizer Nano ZS instrument. The morphology, multilayered structure, lattice fringes of the product were characterized by transmission electron microscopy (TEM) using a JEOL JEM-2010 TEM instrument at an accelerating voltage of $200 \mathrm{kV}$, associated with energy dispersive X-ray spectroscopy (EDS) arrangement for analyzing elemental composition. The tapping mode atomic force microscopy (AFM; Cypher S Oxford instruments) was employed to obtain information about the layered structure. The high resolution AFM imaging was achieved using a Si tip of $10 \mathrm{~nm}$ radius. The three-dimensional images were recorded simultaneously in $512 \times 512$ pixels with $1-2$ $\mathrm{Hz}$ scan rate. The presence of hydroxyl radical $(\mathrm{cOH})$, generated in $\mathrm{Mn} / \mathrm{Co} / \mathrm{Ti} \mathrm{LDH}$ during its use as a photocatalyst was measured with a terephthalic acid (TA) fluorescence probe. The superoxide radicals $\left(\mathrm{O}_{2}{ }^{-}\right)$generated in the $\mathrm{LDH}$ during photocatalysis were investigated by electron paramagnetic resonance (EPR) spectroscopy recorded with a Bruker EMX X-band spectrometer with a field modulation of $100 \mathrm{kHz}$. The photocatalytic reactions were monitored with respect to the dye using a Shimadzu 1800 UV-visible spectrometer. 
The ${ }^{1} \mathrm{H}-\mathrm{NMR}$ analysis of the colorless end products of photodegradation, was done at 800 $\mathrm{MHz}$ with a Bruker Advance spectrometer. A $20 \mathrm{~K}$ signal averaging was followed in ${ }^{1} \mathrm{H}$ NMR experiments with $90^{\circ}$ and other pulses being calibrated using standard methods. The excitation sculpting pulse sequence was effectively used for $\mathrm{H}_{2} \mathrm{O}$ suppression which was obtained directly from Bruker pulse programme library. ${ }^{11}$ The carrier frequency was located on $\mathrm{H}_{2} \mathrm{O}$ resonance for maximizing the suppression of $\mathrm{H}_{2} \mathrm{O}$. A relaxation delay of $2 \mathrm{~s}$ was followed for complete relaxation of $\mathrm{H}_{2} \mathrm{O}$ signal. $\mathrm{D}_{2} \mathrm{O}$ was added to the sample prior to ${ }^{1} \mathrm{H}$ NMR analysis, for signal locking and shimming. The colourless products of degradation were further identified using a Varian 3900 GC-Saturn 2100T GC/MS under column temperature of $45^{\circ} \mathrm{C}(1 \mathrm{~min})$ and $45-250{ }^{\circ} \mathrm{C}\left(5^{\circ} \mathrm{C} \mathrm{min}^{-1}\right.$, hold time: $\left.5 \mathrm{~min}\right)$. On the basis of product identification by ex situ ${ }^{1} \mathrm{H}-\mathrm{NMR}$ and GC-MS analyses, the reaction mechanisms have been proposed for the degradation of the dye. The complete mineralization of the degraded dye metabolite was analyzed by measuring the Total Organic Carbon (TOC) using a TOC analyzer (ANATOC II, SGE International Pty. Ltd., Australia). In order to determine the carbon mass balance associated with degradation, arrangements were made to connect the photocatalytic reactor with the gas chromatograph, ion chromatograph and TOC analyser.

\subsection{Photocatalysis}

The visible light assisted photocatalysis of aqueous Rhodamine B was carried out in a cost effective specially designed photocatalytic device until the dye solution turned colourless.

2.4.1. Photocatalytic device.-The photocatalytic reactions were done in a photocatalytic device comprising of a stainless steel chamber fitted with a tungsten lamp (Philips 38941-1; PS 25, Frost-6100) as a visible light source, equipped with lens $(\lambda>400$ $\mathrm{nm})$ to allow the collimated beam of visible light to be incident onto the reaction mixture, taken in a double walled beaker. The lens is adjusted with respect to the light source, in such a way so as to produce a collimated beam having an intensity of $150 \mathrm{~W} \mathrm{~m}^{-2}$ reaching the reactants present within the beaker. A continuous circulation of water through the outer jacket ensures a constant temperature in the reaction system. The double walled beaker is placed over a magnetic stirrer for vigorous stirring of the reactants. The device is attached to a voltage stabilizer in order to avoid over-voltage and under voltage protection (of specification: high voltage cut-off: 290 V; 240 V output, capacity 1.3 Amps, input 90-290 V range; >95\% efficiency), connected to an AC-source. The core portion photocatalytic device is schematically illustrated in Fig. 2.

2.4.2. Photocatalytic reaction procedure.-The photocatalytic experiments were performed at $30^{\circ} \mathrm{C}$ (room temperature) in a double walled beaker with continuous circulation of running water. In the degradation experiments, the reactants were vigorously stirred for $30 \mathrm{~min}$ in the dark to establish the adsorption-desorption equilibrium between the catalyst and the dye prior to visible light exposure. During this period, the reaction mixture of the test dye was analysed by taking out $5 \mathrm{ml}$ aliquots at every $5 \mathrm{~min}$ intervals and analyzing its centrifugate by UV-vis spectrophotometer. Each photocatalytic reaction was carried out with $200 \mathrm{ml}$ of $10^{-5} \mathrm{M}$ solution of Rhodamine B (Fig. S1; ESI $\dagger$ ). During photocatalysis, at every $10 \mathrm{~min}$ intervals, $5 \mathrm{ml}$ aliquots were taken out, centrifuged to 
remove solid LDH particles and the centrifugate was analyzed for the unconverted dye at $\lambda_{\max }$ of $553 \mathrm{~nm}$. The decolorization efficiency of the dye was evaluated using the relationship $C / C_{O}$ against time, where $C$ and $C_{o}$ represent the absorbance at a particular interval and at $\mathrm{t}=0$ respectively. The decrease in the absorbance of the dye to almost zero after 60 min of visible light exposure indicated the completion of the decolorization process. Control reactions were performed for the dye in the dark for 60 min under the same set of conditions. The effects of $\mathrm{pH}$ on the photocatalytic activities of the test dyes were investigated by variation of the $\mathrm{pH}$ of the reaction mixtures from 4.0 to 11.0 by adding either $0.1 \mathrm{M} \mathrm{HCl}$ or $\mathrm{NaOH}$. Catalyst loadings of 5.0, 10.0, 15.0 and $20.0 \mathrm{mg}$ in $200 \mathrm{ml}$ dye solution was used to investigate the effects of the same on $\mathrm{RhB}$ photo-degradation at $\mathrm{pH}$ 11.0 with dye concentration of $10^{-5} \mathrm{M}$. The photocatalytic conversion was carried out with three concentrations of the dye of $1.0 \times 10^{-3}, 1.0 \times 10^{-4}$ and $1.0 \times 10^{-5} \mathrm{M}$ with LDH loading of $15.0 \mathrm{mg}$ in $200 \mathrm{ml}$ of dye solution separately. The photocatalytic efficiency of $\mathrm{Mn} / \mathrm{Co} / \mathrm{Ti} \mathrm{LDH}$ was compared with that of commercially available pure $\mathrm{MnO}_{2}, \mathrm{CoO}, \mathrm{TiO}_{2}$ and Degussa P25 (15.0 mg in $200 \mathrm{ml}$ of dye solutions) for degradation of $1.0 \times 10^{-5} \mathrm{M}$ dye solution. The kinetics of photodegradation was also evaluated under optimum conditions (concentration $1.0 \times 10^{-5} \mathrm{M}$; catalyst $15.0 \mathrm{mg}$ and $\mathrm{pH} 11,30^{\circ} \mathrm{C}$ ). The photocatalytic efficiency of the LDH was followed separately in the presence of the quenchers like 2NaEDTA ( $\mathrm{h}^{+}$scavenger), $n$-butanol ('OH scavenger), benzoquinone $\left(\mathrm{O}_{2}{ }^{-}\right.$scavenger) added at the beginning of the dye degradation reaction in order to establish the roles played by ${ }^{\circ} \mathrm{OH}, \mathrm{h}$ ${ }^{+}$and $\mathrm{O}_{2}{ }^{-}$species, for establishing the semiconduction mechanism of photocatalysis. The FT-IR data of the colourless end products of dye degradation were compared with those of the pure dyes to evaluate whether adsorption or degradation dominated the decolorization reactions. The colourless end products of photocatalysis were identified by ${ }^{1} \mathrm{H}-\mathrm{NMR}$ and GC-MS techniques for establishing the mechanism of RhB degradation.

\section{Results and discussion}

\subsection{X-ray diffaction study (XRD)}

The powder XRD pattern of the LDH (Fig. 3) under investigation shows the characteristic Bragg reflections of the hexagonal $\mathrm{LDH}$ phase with interlayer $\mathrm{CO}_{3}{ }^{2-}$ ions, similar to those previously reported for the X-ray diffraction data of LDHs. ${ }^{5,7,9,12-14}$ A well-defined series of $(00 I)$ Bragg reflections indicates the presence of parallel layered house-of cards stacking of the LDH nanoparticles. ${ }^{10}$ The presence of (003), (006) and (009) reflections at $2 \theta$ values of $13.34^{\circ}, 26.68^{\circ}$ and $40.02^{\circ}$ respectively indicates the incorporation of $\mathrm{CO}_{3}{ }^{2-}$ ions and $\mathrm{H}_{2} \mathrm{O}$ molecules within the LDH lattice. The d-spacing corresponding to the (003) peak was 0.664 $\mathrm{nm}\left(2 \theta \approx 13.34^{\circ}\right)$ and that of $(110)$ was $0.313 \mathrm{~nm}\left(2 \theta \approx 28.56^{\circ}\right)$. Since the basal spacing of the synthesized LDH is similar to that of Ti containing LDHs, it is likely that the interlayer $\mathrm{CO}_{3}{ }^{2-}$ and $\mathrm{H}_{2} \mathrm{O}$ molecules have retained the usual $\mathrm{LDH}$ pattern. The (110) and (101) peaks at $2 \theta$ values of $28.56^{\circ}$ and $37.43^{\circ}$ indicate the existence of the anatase phase of $\mathrm{TiO}_{2}$ in the synthesized LDH. ${ }^{3}$ The presence of these narrow and sharp diffraction peaks indicate good crystallinity. The other diffraction peaks (018), (100), (0111), (113) and (1013) could also be indexed to typical LDHs. The XRD parameters are illustrated in S2 (ESI $\dagger$ ). 


\subsection{X-ray photoelectron study (XPS)}

The XPS survey spectrum carried out using monochromatic $\mathrm{AlK}_{\mathrm{a}} \mathrm{X}$-ray source reveals the existence of elements in different chemical states on the LDH surface (Fig. 4). Thus, Mn is present in +(III) and +(II) states in the LDH. Ti is present in +(III) and +(IV) whereas Co is present in +(II) and +(III) states. The O 1s XPS scan reveals the existence of the corresponding oxides of different metals associated with the LDH surface. The spectra were deconvoluted to confirm the presence of mixed valency of metals along with the nature of bonding of the constituent elements in the LDH. The presence of mixed valency of metals indicate the occurrence of a series of redox reactions on the LDH surface, being assisted by visible light, which is most likely to degrade the organic dye to colourless simple molecules.

The chemical states of the elements present in the LDH, were identified by comparison of the obtained photoelectron binding energies (BE) with those taken from literature. The full scan photoelectron lines indicate the existence of spin states of exhibited by the elements present within the LDH i.e. Mn 3s, Mn 2p, Co 2p, Ti 2p, O 1s, C 1s, N 1s and Cl 2p respectively. The XPS lines were further deconvoluted to investigate the presence of mixed valency as well as to study the nature of bonding of diffrent groups on the LDH surface. ${ }^{5}$

The magnitude of peak splitting in Mn 3s peak in the LDH is the diagnostic feature of the oxidation state of the metal. This peak on further deconvolution indicates the existence of two multiplet spin splitting, which is likely to have occurred as a result of coupling of nonionized 3s electron with 3d valenceband electrons. The deconvoluted Mn 3s peak [Fig. 4(B)] shows peak splitting corresponding to the binding energy difference of $\Delta E=5.5 \mathrm{eV}$, due to the presence of +(III) oxidation state of Mn. On the other hand, the Mn 2p line could be related to the existence of multiplet splitting due to $\mathrm{Mn} 2 \mathrm{p}_{3 / 2}$ and $\mathrm{Mn} 2 \mathrm{p}_{1 / 2}$, along with their corresponding shake-up satellites [Fig. 4(C)] in the binding energy range of 635-660 eV. These results show that Mn exists in different oxidation states in the LDH. The deconvolution of Mn 2p yielded two peaks and a satellite. The asymmetric peak at $640.7 \mathrm{eV}$ could be attributed to $2 \mathrm{p}_{3 / 2}$ state and the shake-up satellite at $646.8 \mathrm{eV}$ represents $\mathrm{Mn}^{2+}$ state. On the other hand, the deconvoluted peak, at $652.7 \mathrm{eV}$ represents the $2 \mathrm{p}_{1 / 2}$ state of $\mathrm{Mn}$. The splitting between the $2 \mathrm{p}_{3 / 2}$ and $2 \mathrm{p}_{1 / 2}$ XPS lines was $12 \mathrm{eV}$. Thus, the detection of the satellite at $646.8 \mathrm{eV}$ in the $\mathrm{Mn} 2 \mathrm{p}_{3 / 2}$ region accompanied by peak splitting of $\Delta E=5.5$ $\mathrm{eV}$ in $\mathrm{Mn} 3 \mathrm{~s}$ region, proves the existence of $\mathrm{Mn}^{2+}$ and $\mathrm{Mn}^{3+}$ states of the metal. ${ }^{15}$

The core level Co $2 p$ XPS line yielded four peaks corresponding to $2 \mathrm{p}_{3 / 2}$ and $2 \mathrm{p}_{1 / 2}$ spin states of Co and their corresponding satellite lines. ${ }^{15,16}$ The existence of these XPS lines were further confirmed by deconvolution of the Co $2 p$ line, giving two sharp peaks at 781 $\mathrm{eV}$ and $796 \mathrm{eV}$ corresponding to $2 \mathrm{p}_{3 / 2}$ and $2 \mathrm{p}_{1 / 2}$ spin states of Co respectively with a peak splitting of $\Delta E=15 \mathrm{eV}$ representing $\mathrm{Co}^{2+}$ and $\mathrm{Co}^{3+}$ states respectively [Fig. 4(D)]. Meanwhile the low intensity deconvoluted XPS lines at $787 \mathrm{eV}$ and $803 \mathrm{eV}$ represent the associated satellite features of $2 \mathrm{p}_{3 / 2}$ and $2 \mathrm{p}_{1 / 2}$ spin states of Co.

The core level Ti 2p XPS line [Fig. 4(E)] indicates the presence of two asymmetric peaks at binding energies of $456.8 \mathrm{eV}$ and $462.5 \mathrm{eV}$ corresponding to $2 \mathrm{p}_{3 / 2}$ and $2 \mathrm{p}_{1 / 2}$ spin states of Ti. Thus, $\mathrm{Mn} / \mathrm{Co} / \mathrm{Ti} \mathrm{LDH}$ surface is likely to contain of two spin states Ti with a peak separation of $\sim 5.7 \mathrm{eV}$, indicating the presence of $\mathrm{Ti}^{4+}$ species, also confirmed from the XRD 
data. The deconvoluted Ti $2 \mathrm{p}_{3 / 2}$ spin state shows two peaks at $456.5 \mathrm{eV}$ and $457.2 \mathrm{eV}$ indicating the existence of $\mathrm{Ti}^{3+}$ and $\mathrm{Ti}^{4+}$ respectively. The surface transformation of $\mathrm{Ti}$ species also indicates the occurrence of redox reaction at the surface of the LDH. 5,16

The O 1 s core level XPS spectrum of the Mn/Co/Ti LDH consists of a peak at $529.9 \mathrm{eV}$, accompanied by a valley that extends to $536 \mathrm{eV}$. The $\mathrm{O} 1 \mathrm{~s}$ line after deconvolution yields as many as nine XPS peaks [Fig. 4(F)]. The deconvoluted lines at $529.7 \mathrm{eV}$ and $529.9 \mathrm{eV}$ are likely to correspond to the existence of $\mathrm{Ti}_{2} \mathrm{O}_{3}$ and $\mathrm{TiO}_{2}$ species on the $\mathrm{LDH}$ surface. The lines at $530.2 \mathrm{eV}$ and $530.4 \mathrm{eV}$ could be attributed to $\mathrm{MnO}$ and $\mathrm{Mn}_{2} \mathrm{O}_{3}$ species whereas that observed at $530.7 \mathrm{eV}$ and $531 \mathrm{eV}$ could be assigned to the presence of $\mathrm{CoO}$ and $\mathrm{Co}_{2} \mathrm{O}_{3}$ species respectively. The line at $531.3 \mathrm{eV}$ could be due to the existence of physisorbed $\mathrm{H}_{2} \mathrm{O}$ or $-\mathrm{OH}$ groups present on the $\mathrm{LDH}$ surface. $\mathrm{O} 1 \mathrm{~s}$ line at $532 \mathrm{eV}$ could be attributed to $\mathrm{C}=\mathrm{O}$ group of the interlayer $\mathrm{CO}_{3}{ }^{2-}$ species and the very low intensity line at $535.2 \mathrm{eV}$ could be due to $\mathrm{NO}_{3}{ }^{-}$species, arising from the use of nitrate salts of $\mathrm{Mn}$ and $\mathrm{Co}$, as precursors in the synthesis of $\mathrm{Mn} / \mathrm{Co} / \mathrm{Ti} \mathrm{LDH}^{15,16}$

The C 1s XPS line [Fig. 4(G)] appears at $288 \mathrm{eV}$ and its corresponding satellite feature is observed at $289.4 \mathrm{eV}$. On deconvolution, lines at $288.1 \mathrm{eV}$ and $289.5 \mathrm{eV}$ are obtained, which could be attributed to $\mathrm{C}=\mathrm{O}$ and $\mathrm{O}=\mathrm{C}-\mathrm{O}$ groups associated with the interlayer $\mathrm{CO}_{3}{ }^{2-}$ species. ${ }^{5,16}$ The core level $\mathrm{N} 1 \mathrm{~s}$ XPS line is highly sensitive to the chemical environment of nitrogen and varies from 396 to $410 \mathrm{eV}$ in the LDH [Fig. 4(H)]. The peak at BE of $402.9 \mathrm{eV}$ associated with a satellite at $405 \mathrm{eV}$ that extends upto $409 \mathrm{eV}$ is due to $\mathrm{N}_{2}$ species associated with the LDH surface. Deconvolution of N 1s line yields three peaks at BE values of 402.8 $\mathrm{eV}, 405 \mathrm{eV}$ and $406.8 \mathrm{eV}$ respectively. The first two of these could be attributed to well screened and poorly screened terminally bonded $\gamma-\mathrm{N}_{2}$ species respectively. The low intensity peak at $406.8 \mathrm{eV}$ is likely to be the satellite of $\mathrm{N} 1 \mathrm{~s}$ peak. N 1s XPS peaks could be attributed to the use of urea as the basic precipitant in LDH synthesis. ${ }^{15,16}$

$\mathrm{Cl} 2 \mathrm{p}$ XPS line is composed of two peaks corresponding to $2 \mathrm{p}_{3 / 2}$ and $2 \mathrm{p}_{1 / 2}$ spin states at binding energies of $198.3 \mathrm{eV}$ and $199.4 \mathrm{eV}$ respectively [Fig. 4(I)]. The peak at $198.3 \mathrm{eV}$ indicates the presence of $\mathrm{Cl} 2 \mathrm{p}_{3 / 2}$ state, and that observed at $199.4 \mathrm{eV}$ is due to $2 \mathrm{p}_{1 / 2}$ spin state of $\mathrm{Cl}$. The $\mathrm{Cl} 2 \mathrm{p}$ core level XPS lines in the LDH could have arisen due to the use of $\mathrm{TiCl}_{4}$ as a precursor of Ti during the $\mathrm{LDH}$ synthesis. ${ }^{16}$

\subsection{FT-IR, EIS and TG analyses}

The existence of interlayer $\mathrm{H}_{2} \mathrm{O}$ molecules and $\mathrm{CO}_{3}{ }^{2-}$ anions in the interlayer galleries of the LDH could be confirmed further from the FT-IR spectra [S3; Fig. S2 (ESI $\dagger)$ ]. The absorption band at $3440 \mathrm{~cm}^{-1}$ has been attributed to the stretching vibrations associated with the surface and interlayer $\mathrm{H}_{2} \mathrm{O}$ molecules and $\mathrm{OH}^{-}$groups, being observed at a lower frequency in $\mathrm{Mn} / \mathrm{Co} / \mathrm{Ti} \mathrm{LDH}$ than that of free water at $3600 \mathrm{~cm}^{-1}$. This may be considered as indicating the existence of interlayer $\mathrm{H}_{2} \mathrm{O}$ in the $\mathrm{LDH}$. The shoulders at $3178 \mathrm{~cm}^{-1}$ and $2936 \mathrm{~cm}^{-1}$ could be attributed to the $\mathrm{H}-$-bonding between interlayer $\mathrm{H}_{2} \mathrm{O}$ and $\mathrm{CO}_{3}{ }^{2-}$ anions. The band at $1638 \mathrm{~cm}^{-1}$ is indexed to the bending vibrations of $\mathrm{H}_{2} \mathrm{O}$ molecules. Three infrared active vibrations associated with the interlayer $\mathrm{CO}_{3}{ }^{2-}$ species are observed in this $\mathrm{LDH}$, at $1412 \mathrm{~cm}^{-1}\left(\mathrm{n}_{3}\right), 1128 \mathrm{~cm}^{-1}\left(\mathrm{n}_{1}\right)$ and $721 \mathrm{~cm}^{-1}\left(\mathrm{n}_{2}\right)$. Apart from these vibrations, the bands at $866 \mathrm{~cm}^{-1}$ and $630 \mathrm{~cm}^{-1}$ could be indexed to $\mathrm{M}-\mathrm{OH}$ and $\mathrm{M}-\mathrm{O}$ stretching vibrations. 
The band at $866 \mathrm{~cm}^{-1}$ could be attributed to in-plane quadrant bending and that at $1128 \mathrm{~cm}$

${ }^{-1}$ to $\mathrm{C}-\mathrm{O}$ stretching. Thus, the IR results further confirm the existence of $\mathrm{CO}_{3}{ }^{2-}$ ions and $\mathrm{H}_{2} \mathrm{O}$ molecules in the interlayer galleries of the LDH. ${ }^{5,13}$

The electrochemical impedance spectroscopy (EIS) of the LDH was done with $2.5 \mathrm{mM}$ $\mathrm{K}_{3}\left[\mathrm{Fe}(\mathrm{CN})_{6}\right] / \mathrm{K}_{4}\left[\mathrm{Fe}(\mathrm{CN})_{6}\right](1: 1)$ mixture in a $0.5 \mathrm{M}$ aqueous solution of $\mathrm{Na}_{2} \mathrm{SO}_{4}$, acting as redox probe. The narrow arc radius of the Nyquist impedance plot [S4; Fig. S3(A); ESI $\dagger$ ] represents a decrease in solid state interfacial layer resistance, accompanied by a high charge transfer effciency of the LDH electrode. Thus, a rapid charge carrier transport could be achieved with an effective separation, thereby facilitating the semiconduction mechanism. From this, it could be understood that the electron transporting properties of the LDH is facilitated by suppression of $\mathrm{e}^{-}-\mathrm{h}^{+}$pair recombination. ${ }^{16}$

The Mott-Sckottky impedance analysis exhibited a reversed sigmoid curve for the LDH indicating n-type semiconductor properties to the LDH [S4; Fig. S3(B); ESI $\dagger$ ]. The flat band potential, $\left(\mathrm{V}_{\mathrm{fb}}\right)$, obtained from the $\mathrm{X}$-intercept of the linear region of the Mott Sckottky plot, showing a negative value indicating the presence of different surface states that could produce considerable changes in the band positions. $\mathrm{V}_{\mathrm{fb}}$ for $\mathrm{Mn} / \mathrm{Co} / \mathrm{Ti} \mathrm{LDH}$ electrode was observed at $-0.77 \mathrm{~V}$ vs. SCE; which is equivalent to $0.54 \mathrm{~V}$ vs. NHE. This suggests the existence of different surface states within the LDH which has lead to a considerable change in the band positions. The occurrence of negative flat band with different surface states is explained on the basis of Fermi-level pinning. ${ }^{17}$ In the absence of redox couple, flat band potential $\left(V_{\mathrm{fb}}\right)$ corresponds to the energy very close to the conduction band edge $\left(\mathrm{E}_{\mathrm{c}}\right)$ of a surface state free semiconductor [S4; Fig. S3(C); ESI $\dagger$ ]. In the absence of any specific adsorption, the flat band potential is found to drop across the Helmholtz layer, $\Delta \varphi_{\mathrm{H}}$; corresponding to the potential attributable to the dipoles oriented at the electrode interface, $\mathrm{V}_{\mathrm{d}}$; whereas, the potential associated with the space-charge region of the semiconductor electrode, $\Delta \varphi_{\mathrm{SC}}$, is zero.

In order to carry out the impedance measurements, an electrolyte of moderate concentration is used for neglecting the potential drop across the diffuse double layer associated with the liquid-LDH electrode interface. On varying the potential difference between the LDH electrode and the solution, by application of an external potential at $E_{\text {redox }}=e V_{\text {redox }}$ in solution, $\Delta \varphi_{\mathrm{H}}$ remains unchanged, while $\Delta \varphi_{\mathrm{SC}}$, remains equal to the applied potential (assuming no specific adsorption of redox couple and no change in $\mathrm{V}_{\mathrm{d}}$ ). This forms the basis of the usual semiconduction model, wherein, the band energies are most likely to remain fixed [i.e. $\Delta\left(\Delta \varphi_{\mathrm{H}}\right) \approx 0$ ], the open circuit photopotential $(\Delta \mathrm{V})$ is represented by the equation-

$$
\Delta V=\left|\Delta V_{\mathrm{fb}}^{\circ}-V_{\text {redox }}\right|
$$

However, no photoresponse is expected for redox couples possessing energies beyond the band gap energy of the semiconductor electrode. Thus, eqn (1) forms the basis of selection of redox couples with potential corresponding to the n-type semiconductor, for maximizing the output voltage of a photochemical cell. On the other hand, the flat band potential is 
shifted by adsorption of specific ions, since a drop in potential across the Helmholtz layer, in presence of surface charge, $\mathrm{q}_{\mathrm{s}}$, is found to occur and is represented by eqn (2) as-

$$
\Delta \varphi_{\mathrm{H}}=\frac{q_{\mathrm{s}}}{C_{\mathrm{H}}}+V_{\mathrm{d}}=\frac{q_{\mathrm{s}}}{\varepsilon \varepsilon_{0} / d}+V_{\mathrm{d}}
$$

where $\mathrm{C}_{\mathrm{H}}$ represents the Helmholtz layer capacitance, $\boldsymbol{\varepsilon}$ is the dielectric constant, $\varepsilon_{0}$ represents the permittivity of free space and $d$ is the Helmholtz layer thickness. Thus, adsorption of the anion $\left(\mathrm{q}_{\mathrm{s}}<0\right)$ has led to a negative shift in $\mathrm{V}_{\mathrm{fb}}$ potential, for the Fe/Ti LDH electrode. The overall filling or emptying of surface states results in the production of surface charge, which is a function of the number of states with respective occupancies. According to eqn (1), $\mathrm{V}_{\mathrm{fb}}$ will be shifted, producing a relative location of the conduction and valence band edges with respect to the energy level of the redox couple of the solution. As the surface state density of the LDH is very large, a change in potential between the solution and the LDH, will impart an equal change in $\Delta \varphi_{\mathrm{H}}$, keeping $\Delta \varphi_{\mathrm{SC}}$ constant. Under the set of conditions, Fermi level pinning occurs for the energy level of the surface state $\left(\mathrm{E}_{\mathrm{Ss}}\right)$ and the photopotential remain independent of $\mathrm{V}_{\text {redox }}$. As pinning exists, photoactivity may be observed for redox couples having potentials located apparently outside the energy gap region (depending on $V_{\mathrm{fb}}^{\circ}$ value) of the semiconductor [S4; Fig. S3(D); ESI $\dagger$ ]. Moreover, the transport properties of the LDH also occurs due to the negative flat-band potential having higher migration efficiency of charge carriers. Generally, the conduction band potential $\left(\mathrm{E}_{\mathrm{CB}}\right)$ of $\mathrm{n}$-type semiconductors lies between 0 to $-0.2 \mathrm{~V}$. In this case, $\mathrm{V}_{\mathrm{fb}}$ falls within the range of n-type semiconductors, depending on the effective electronic mass and carrier concentration. In impedance measurements, the voltage difference between $\mathrm{E}_{\mathrm{CB}}$ and $\mathrm{V}_{\mathrm{fb}}$ is set at $0.1 \mathrm{~V}$. The negative Fermi level of the $\mathrm{LDH}$ makes $\mathrm{E}_{\mathrm{CB}}$ to lie at $-0.54 \mathrm{~V}$ vs. NHE (i.e. $-0.77 \mathrm{~V}$ vs. SCE). This imparts a strong reducing power to the LDH involved in the oxygen reduction reaction. The standard redox potential of $\mathrm{O}_{2} / \mathrm{O}_{2}{ }^{--}$couple is $-0.28 \mathrm{~V}$ vs. NHE, a comparatively high positive value than the observed $\mathrm{E}_{\mathrm{CB}}$ for the $\mathrm{LDH}$. This suggests that the electrons photogenerated by the $\mathrm{LDH}$ could interact with the adsorbed $\mathrm{O}_{2}$ species forming $\mathrm{O}_{2}{ }^{--}$on the LDH surface. The EIS measurements suggests that photogenerated electrons on the LDH surface, leaves hole type charge carriers which promotes the photodegradation of the dyes.

The TG analysis was performed to investigate the thermal stability of the LDH. Three degradation steps could be observed for the LDH [S4, Fig. S3(E); ESI †]. Weight loss between $70-135^{\circ} \mathrm{C}$ with a peak at $100{ }^{\circ} \mathrm{C}$, indicated by the DTG curve, could be assigned to the loss of physisorbed and interlayer water constituting $4 \%$ by weight of the material. The second loss, observed from $244-335^{\circ} \mathrm{C}$ with a sharp peak at $310{ }^{\circ} \mathrm{C}$, accounted for $14.5 \%$ weight loss, and was observed due to the dehydroxylation of the brucite like layers. The third weight loss of $11.5 \%$ from $339-460{ }^{\circ} \mathrm{C}$, associated with a broad peak at $372{ }^{\circ} \mathrm{C}$, was likely to have occurred due to the decomposition of the interlayer $\mathrm{CO}_{3}{ }^{2-}$ ions. ${ }^{17,18}$ No further weight loss was observed above $460{ }^{\circ} \mathrm{C}$, which indicated the presence of very negligible amount of carbonaceous residues in the LDH. The thermogravimetric also 
supported the existence of $\mathrm{H}_{2} \mathrm{O}$ and $\mathrm{CO}_{3}{ }^{2-}$ ions in the interlayer galleries of the layered material.

\subsection{Optical properties}

The UV-visible DRS and photoluminescence studies of the LDH provide an insight into absorption and emission processes on the LDH surface. The life time of the charge carriers could be evaluated by time resolved photoluminescence emission analysis, as discussed in the following sections-

3.4.1. UV-visible diffuse reflectance spectra (DRS).-UV-vis DRS measurements could be utilized to ascertain the nature and coordination states of $\mathrm{Mn}$, Co and Ti in the layered framework [Fig. 5(A)]. The hump at $\sim 230 \mathrm{~nm}$ could be attributed to d-d transitions. The strong absorption band in the region of $250-460 \mathrm{~nm}$ could be assigned to typical M(II) co-ordinated to $\mathrm{CO}_{3}{ }^{2-}$ in the interlayer galleries. The broad shoulder observed between 460 to $700 \mathrm{~nm}$ indicates the presence of $\mathrm{Ti}^{4+}$ in the brucite like sheets. The broad valley signifies a supramolecular guest-guest H-bonding, van der Waals forces or guest-host interactions. Compared to the commercial catalysts, a band broadening accompanied by a red shift is observed for the $\mathrm{LDH}$, due to the formation of aggregates. A comparison was done to evaluate the nature of UV-visible absorption of the LDH over commercial catalysts like $\mathrm{MnO}_{2}, \mathrm{CoO}, \mathrm{TiO}_{2}$, and Degussa P25. The DRS data show strong absorbance at 250-448 nm for all the commercial catalysts [Fig. 5(A)]. The LDH exhibited stronger absorption between 460-700 nm, which was not observed in any of the commercial catalysts. The stronger absorbance of the LDH in the visible region shows its potential for dye degradation over commercial catalysts. ${ }^{7,13}$

3.4.2. Band gap energy.-A comparative analysis of band gap energy [Fig. 5(B)] is performed to have a better understanding of the optical properties of the LDH over the commercial catalysts; following the classical Tauc equation represented by (eqn 3):

$$
\varepsilon h v=K\left(h v-E_{g}\right)^{n}
$$

wherein, $\mathrm{E}_{\mathrm{g}}$ represents the optical band gap, hn is the photon energy, $\mathrm{K}$ is a proportionality constant, 3 is the absorption coefficient and $\mathrm{n}$ depends on the nature of the transition. The value associated with the point of intersection of the line tangent to the plotted curve inflection point with the horizontal line (hn axis) is the band gap $\mathrm{E}_{\mathrm{g}}$ value. The best fit of $(\varepsilon h v)^{2}$ versus hn plots was obtained for $\mathrm{n}=1 / 2$, suggesting a direct allowed transition across the energy band gap. The absorption edge energies corresponding to $\mathrm{E}_{\mathrm{g}}=2.62 \mathrm{eV}$ was observed for the LDH; this band transition is in good agreement with the photoluminescence observations of the LDH. The respective band gap energies of the commercial catalysts are: $\mathrm{MnO}_{2}(2.5 \mathrm{eV}), \mathrm{CoO}(2.68 \mathrm{eV}), \mathrm{TiO}_{2}(3.26 \mathrm{eV})$ and Degussa P25 (3.17 eV). Excepting $\mathrm{MnO}_{2}$, the $\mathrm{LDH}$ has been found to possess a narrow band gap in comparison to that of the other commercial catalysts. Thus, a higher photoactivity is associated with the LDH. The red shift observed in the UV-Vis DRS of the LDH could be attributed to the narrow band gap of the LDH. ${ }^{16}$ The band gap in the LDH could have been reduced due to the presence of 
localized gap states induced by doping with $\mathrm{Ti}^{4+}$ accompanied by oxygen vacancies within the layered framework.

3.4.3. Photoluminescence (PL) emission measurements.- In order to have an insight into the effects of recombination of charge carriers, photoluminescence (PL) analysis was carried out at room temperature $\left(30^{\circ} \mathrm{C}\right)$. The luminescence emissions are classified into two categories: band-band PL emission belonging to the electronic transitions from the conduction band (CB) bottom to the valence band (VB) top and the excitonic PL emissions that arise from the surface oxygen vacancies and defects associated with the semiconductor. $16,18,19$ In case of band-band PL emission, lower the luminescence intensity, higher is the separation rate of photo-induced electron $\left(\mathrm{e}_{\mathrm{CB}}{ }^{-}\right)$- hole $\left(\mathrm{h}_{\mathrm{vb}}{ }^{+}\right)$pair; therefore higher is its photoactivity. In case of excitonic emissions, the relationship between luminescence intensity and photoactivity is complicated to a considerable extent and depends primarily on the electronic properties of semiconductors.

In this work, the luminescence emissions of the LDH were performed at excitation wavelengths of $350 \mathrm{~nm}, 380 \mathrm{~nm}$ and $410 \mathrm{~nm}$ respectively [Fig. 5(C)]. The luminescence intensity was found to decrease on increasing the excitation wavelength, which could be attributed to higher separation of the charge carriers, consistent with the electrochemical impedance results. The oxygen vacancies and defects bind the photo-induced electrons to form excitons, so that the luminescence signal can easily occur. It is known that Ti doped photocatalytic systems consist of two kinds of trap sites for holes, (i) the deep hole and (ii) the shallow hole. The deep trapped holes exhibit a lower oxidizing potential and mostly prefer to interact with physisorbed molecules. The LDH showed several excitonic PL emissions at $448 \mathrm{~nm}(2.77 \mathrm{eV}), 473 \mathrm{~nm}(2.62 \mathrm{eV}), 531(2.34 \mathrm{eV}), 550 \mathrm{~nm}(2.25 \mathrm{eV})$ and 581 $\mathrm{nm}(2.13 \mathrm{eV})$ accompanied by a valley extending upto $700 \mathrm{~nm}$ respectively, resulting from the surface oxygen vacancies and defects present in $\mathrm{Mn} / \mathrm{Co} / \mathrm{Ti} \mathrm{LDH}$. These are suggested to be responsible for the significant enhancement in the optical properties of the LDH. The UV emission peak at $473 \mathrm{~nm}(2.62 \mathrm{eV})$ could be attributed to the exciton emissions from the conduction band $\left(\mathrm{e}_{\mathrm{CB}}{ }^{-}\right)$to the valence band $\left(\mathrm{h}_{\mathrm{VB}}{ }^{+}\right)$. This is consistent with the band gap energy of $\mathrm{Mn} / \mathrm{Co} / \mathrm{Ti} \mathrm{LDH}$. Apart from the above, other emissions could also be observed and these are mostly associated with excitons and oxygen defects in the shallow and deep trap centers.

The emission peak at $448 \mathrm{~nm}$ is due to self-trapped exciton (STE). A STE is generated on the lattice site on a hole capture by a trapped electron, which is of two types, direct and indirect. A direct STE occurs due to direct recombination of charge carriers, while in an indirect STE, the recombination occurs via oxygen vacancies. Since Ti doping generates oxygen vacancy, the STE peak at $448 \mathrm{~nm}$ is most likely to have occurred via oxygen vacancy and is of indirect nature. The emission peak at $473 \mathrm{~nm}$ is due to the F-center present within the LDH. The F center is an oxygen vacancy with two trapped electrons. The emissions associated with oxygen defects occur as a result of the presence of oxygen vacancy in the shallow or deep levels within the band gap of the LDH. However, oxygen defects of the grain boundary of the LDH are charged, which are distributed randomly and acts as shallow trap levels. The observed luminescence peaks at $448 \mathrm{~nm}, 531 \mathrm{~nm}, 550 \mathrm{~nm}$ and $587 \mathrm{~nm}$ could 
be attributed to the shallow trap levels. However, the shoulders at $630 \mathrm{~nm}$ extending upto $700 \mathrm{~nm}$ could be assigned to the deep trap emissions.

The free electrons which are untrapped by the defects, migrate most likely throughout the lattice and undergo recombination with the holes on the LDH surface. The migrating electrons are likely to be repelled by the charged oxygen vacancies preventing further recombination with holes. As defect states arise due to doping, the mobility of the charge carriers is thus slowed down, which delays the recombination process. Moreover, the dopants present on the LDH surface obstruct the efficient overlap of charge carriers across the LDH surface. The d-states of the LDH act as active trap centers and separate the charge carriers. Besides this, doping causes shifting of the band edges and hence the electrons of the conduction band have to traverse an indirect path for undergoing recombination with the holes and quench the emission intensity. As the emission intensity is reduced due to increase in excitation wavelength, the quenching effect is most likely to play an important part in the reduction of the emission intensity. Doping of ions increases the interaction between the dopant and defects; thereby forming quenching centers. The electrons present within the $\mathrm{LDH}$, are most likely to be captured at first by shallow trap levels from which they jump to $\mathrm{Mn}, \mathrm{Co}$ or Ti d-states, which may further jump from one d-state to another and thus forms a number of quenching centers. The electrons, however, after passing through a channel of dopant and defects, ultimately undergo recombination with the holes and reduce the emission intensity. Thus, the presence of defects within the microstructures of the LDH is most likely to play a major role in its high photoactivity under visible light.

3.4.4. Photoluminescence excitation spectra.-The photoluminescence excitation spectra of the LDH was monitored at different excitation wavelengths in order to identify the accuracy of excitation luminescence wavelengths. The results are illustrated in Fig. 5(D).

The excitation spectra showed strong peaks at $350,380,410$ and $528 \mathrm{~nm}$; on being excited at the emission wavelengths of 450,475 and $580 \mathrm{~nm}$ respectively. The results validate the excitation wavelengths used for observation of the luminescence properties. Meanwhile a shift in the luminescence peak from 380 to $384 \mathrm{~nm}$, marked by an increase in intensity at $580 \mathrm{~nm}$ emission wavelength, indicates the existence of multiple luminescent centres in the LDH.

3.4.5. Time resolved emission study (TRES).- - The dynamics associated with the photo-generated charge carriers has been evaluated further using time-resolved photoluminescence emission spectroscopy (TRES), the instrumentation was based on a streak camera which enables the calculation of the temporal decay time of the carriers. ${ }^{19}$ The luminescence data have been fitted on the two-exponential function equation-

$$
I(t)=A_{1} \exp \left(\frac{t}{\tau_{1}}\right)+A_{2} \exp \left(\frac{t}{\tau_{2}}\right)
$$

where $\tau_{1}, \tau_{2}$ represents the decay times of the fast and slow components; $A_{1}, A_{2}$ are the corresponding luminescence amplitudes. $\tau_{2}$ is associated with indirect formation of selftrapped excitons generated by trapped electrons whereas $\tau_{1}$ is due to the direct formation of 
free electrons and holes. The typical luminescence lifetime fitting results are shown in Fig. 5(E) and Table 1. The longer luminescence decay time for the LDH corresponding to excitation at $410 \mathrm{~nm}$ could be attributed to the delay in radiative recombination process of the charge carriers. Chi-square $\left(x^{2}\right)$ fitting reveals $x^{2}$ values of $\sim 1$, for the decay curves, representing the accuracy and validity of the data fit. The longer luminescence lifetimes of the charge carriers impart high photoactivity to the LDH for undergoing reactions with the target molecules (dyes). It is observed that the average lifetime of the photo-generated charge carriers of $2: 1: 1 \mathrm{Mn} / \mathrm{Co} / \mathrm{Ti} \mathrm{LDH}$ at $410 \mathrm{~nm}$ excitation wavelength is $5.68 \mathrm{~ns}$, which is 1.24 and 2.07 times longer than that observed at $350 \mathrm{~nm}$ and $380 \mathrm{~nm}$ excitation wavelengths. The photo-excited electrons or holes with a longer life time, being generated by photons of wavelength in the visible region on the LDH surface are likely to play an important role in the decomposition of the dye.

3.4.6. Mechanism of photoluminescence.-The luminescence in the LDH could be interpreted on the basis of the results obtained from the luminescence spectra, schematically illustrated in Fig. 5(F). It is observed that the LDH on being excited at 350, 380 and $410 \mathrm{~nm}$ respectively above its band gap energy, a part of the energy is consumed for promoting the electrons from the valence band (VB) to the conduction band (CB), leaving holes in the VB, and the remaining part is used to impart kinetic energy to the electrons, when some of them are likely to jump to the conduction band maximum $\left(\mathrm{CB}_{\max }\right)$. In this, they are most likely to undergo non-radiative transition by hopping to the bottom of the conduction band (Step 1) and may undergo direct UV emission (Step 2). Some of these electrons are likely to be trapped by shallow and deep traps and emit light (Steps 3 and 4). The electrons are then likely to hop to Mn, Co or Ti d-states and recombine with the holes, present in the valence band (Step 5). This process however delays the recombination process as is evident from the lifetimes of the carrier species, revealed by TRES analysis. Thus, the semiconductor LDH, possessing defects and oxygen vacancies within the layered framework, could be a highly efficient photocatalyst for degradation of dyestuffs.

\section{5. $\mathrm{N}_{2}$ adsorption-desorption and dynamic light scattering (DLS) measurements}

$\mathrm{N}_{2}$ adsorption-desorption measurements at $77 \mathrm{~K}$ yielded a Type IV isotherm [S5; Fig. S4(A); ESI $\dagger$ ] with a H3-type hysteresis loop $\left(\mathrm{P} / \mathrm{P}_{0}>0.4-0.8\right)$, indicating that the condensation of $\mathrm{N}_{2}$ within the pores of the $\mathrm{LDH}$ and its release at reduced pressures followed different paths; a characteristic of mesoporous materials. The specific surface area of $190 \mathrm{~m}^{2} \mathrm{~g}^{-1}$ with broad pore width of $3.86 \mathrm{~nm}$ [S5; Fig. S4(B); ESI $\dagger$ ] could be determined for the LDH. The large surface area and pore width could be attributed to the use of urea as a basic precipitant during LDH synthesis, and the LDH is dominated by both micropores and mesopores having diameters from 1-6 nm. The adsorption-desorption cumulative pore volume was 0.218 and $0.201 \mathrm{~cm}^{3} \mathrm{~g}^{-1}$ respectively. Thus the $\mathrm{LDH}$ with an appropriate spatial array of pores within its layered framework, is most likely to assist the electron-hole transfer process responsible for its highly efficient photocatalytic performance. ${ }^{20-22}$

The dynamic light scattering measurements with the LDH were done in aqueous medium in the $\mathrm{pH}$ range of 4-11 for determining the electrokinetic potential ( $\xi$-potential) [S5; Fig. $\mathrm{S} 4(\mathrm{C})$; ESI $\dagger$ ]. A monotonic decrease of surface charge with $\mathrm{pH}$ was observed having a zero 
point charge occurring at $\mathrm{pH}$ 7.16; which is the isoelectric point of the LDH. ${ }^{16}$ Thus, the cationic $\mathrm{RhB}$ dye is most likely to adsorb at $\mathrm{pH}>\mathrm{pH}_{\mathrm{zpc}}$ when the surface attains negative charge. The surface charge was observed to be -31.53 at $\mathrm{pH} 11$ suggesting a maximum degra dation at this $\mathrm{pH}$.

\subsection{Morphology and elemental composition}

The morphology, lattice fringe separation and multilayered structure of the LDH were studied with TEM and AFM measurements. The elemental composition was estimated with EDX.

3.6.1. TEM results.-TEM measurements revealed ultra-fine hexagonally stacked nanoparticles one over the other [Fig. 6(A and C)]. The SAED pattern [Fig. 6(B)] exhibited a hexagonal arrangement of the diffraction peaks as expected for the in-plane symmetry intrinsic to LDH layers, showing the house-of-cards type of stacking of the hetero-layered LDH crystallites. The distance between the periodic lattice fringes from (110) plane of the LDH [Fig. 6(D)] was calculated to be $0.313 \mathrm{~nm}$, matching the value obtained from XRD [S2; Table S1 (ESI $\dagger$ )]. The average particle size was found to be $118 \mathrm{~nm}$, calculated using the histogram plot [Fig. 6(E)]. The cross sectional TEM image [Fig. 6(G and H)] was utilized to obtain the distance between two consecutive parallel metal hydroxide layers to be $0.671 \mathrm{~nm}(=0.289+0.382 \mathrm{~nm})$ from the correlation between the cross sectional TEM image and the idealized LDH structure [Fig. 6(I)]. This is in agreement with the $c^{\prime}$ lattice parameter, obtained from XRD [S2; Table S2 (ESI $\dagger$ )]. Thus, the existence of parallel house of cards type stacking of brucite sheets, exhibiting multilayered structure, hexagonal platelets and periodic lattice fringe separation of diffraction planes, could be confirmed in good agreement with the previous reports of LDH materials. $5,16,19,21,22$

\subsubsection{Energy dispersive X-ray spectroscopy (EDX) results.-The EDX} measurements [Fig. 6(F)] showed that the atomic/ weight ratio of $\mathrm{Mn} / \mathrm{Co} / \mathrm{Ti}$ in the $\mathrm{LDH}$ was $2.06: 1.04: 1$ matching the stoichiometric $\mathrm{Mn} / \mathrm{Co} / \mathrm{Ti}$ ratio of $2: 1: 1$ in the synthesized $\mathrm{LDH}$. The presence of $\mathrm{Cl}, \mathrm{C}$ and $\mathrm{N}$ in the EDX spectra is due to the use of $\mathrm{TiCl}_{4}$ as precursor of $\mathrm{Ti}$ and urea as the basic precipitant during the hydrothermal synthesis. The elemental composition obtained from EDX is presented in Table 2.

3.6.3. Atomic force microscopy (AFM) measurements.-The atomic force microscopy analysis provided further insight into the morphology, thickness and multilayered structure of the LDH [Fig. 7(A)]. The AFM confirms the presence of hexagonal platelets having internal angles around $120^{\circ}$, consistent with the TEM results. ${ }^{16}$ The flat crystallite terrace [Fig. 7(B and C)] of the height profiles provide a strong evidence for the presence of parallel brucite sheets, which are aligned parallel to each other in the substrate to maximize the attractive energy.

The total thickness of Mn/Co/Ti LDH along the $c$-axis [Fig. 7(C)] is determined to be $24 \mathrm{~nm}$ from the 3D height profile of the AFM image. The thickness of a single brucite sheet is found to be $0.382 \mathrm{~nm}$ from the height profile [Fig. 7(B)] in good agreement with cross 
sectional TEM results. On dividing the total thickness with $\mathrm{c}^{\prime}$ lattice parameter (determined from XRD), it could be found that the LDH consists of $\sim 35-36$ parallel brucite sheets.

\subsection{Photocatalysis reactions}

During the 30 min time allowed for reaching the adsorption- desorption equilibrium in dark, aliquots were taken out every $5 \mathrm{~min}$, centrifuged to remove the solid catalyst particles, if any and the centrifugate was analysed for the dye by UV-vis spectrophotometer. No change in the concentration of the dye was observed illustrating absence of adsorption/degradation on the surface of the LDH in the dark prior to light irradiation and also reaching adsorptiondesorption equilibrium [S6; Fig. S5 (ESI $\dagger$ )]. After this period, the reactants were subjected to visible light irradiation, and at every $10 \mathrm{~min}$ intervals, $5 \mathrm{ml}$ aliquots were taken out, centrifuged, and the centrifugate was analyzed for the dye at $\lambda_{\max }$ of $553 \mathrm{~nm}$ for $\mathrm{RhB}$. The decolorization efficiency of the LDH was evaluated by plotting $C / C_{O}$ against time ( $C$ and $C_{O}$ correspond to dye concentration after a time interval and at $t=0$, equivalent to the absorbances of the aqueous solution at a particular interval and at $t=0$ respectively). The absorbance due to the dye decreases to almost zero after $60 \mathrm{~min}$ of light exposure indicating completion of the decolorization process. The solution colour changed to nearly transparent marking the end of the photocatalytic degradation of the dye [Fig. 8(A and B)].

Control experiments performed under the same conditions, but without visible light irradiation, yielded negligible degradation, indicating that visible light plays a dominant role in the reactions. $\mathrm{LDH}$ amount, initial dye concentration and $\mathrm{pH}$ were also varied to study their effects on the degradation of the dye. The photocatalytic efficiency of the LDH was studied separately by addition of quenchers, $2 \mathrm{Na}$-EDTA $\left(\mathrm{h}^{+}\right.$scavenger), $n$-butanol ( ${ }^{\circ} \mathrm{OH}$ scavenger), benzoquinone $\left(\mathrm{O}_{2}{ }^{\cdot-}\right.$ scavenger $)$ at the beginning of the reaction in order to establish the role of ${ }^{\bullet} \mathrm{OH}, \mathrm{h}^{+}$and $\mathrm{O}_{2}{ }^{--}$on photodegradation.

The presence of hydroxyl radical ( $\mathrm{OH})$, generated by the LDH during photocatalysis was further confirmed by using a terephthalic acid (TA) fluorescence probe. The superoxide radical $\left(\mathrm{O}_{2}{ }^{-}\right)$generated by the LDH during photocatalysis was also investigated by electron paramagnetic resonance (EPR) spectroscopy, recorded on a Bruker EMX X-band spectrometer with a field modulation of $100 \mathrm{kHz}$. The photostability of the LDH was justified by its comparative FT-IR analysis and that recovered after the fifth catalytic cycle. The colourless end products were compared with the pure dye to evaluate the occurrence of adsorption or degradation during the photocatalytic process. Insights obtained from ex situ ${ }^{1} \mathrm{H}-\mathrm{NMR}$ and GC-MS analyses of the colorless end products of Rhodamine B degradation were used for derivation of the reaction mechanism.

3.7.1. Effects of LDH amount.-LDH amount was varied from $5.0-20.0 \mathrm{mg}$ (in 200 $\mathrm{ml}$ of the dye solution of concentration $1 \times 10^{-5} \mathrm{M}$ ) and $C / C_{o}$ rs. irradiation time was plotted as in Fig. 9(A). The decolorization efficiency increased with LDH amount. RhB degraded $\sim 99.8 \%$ at $15.0 \mathrm{mg}$ of LDH. A larger amount increases the available surface area and consequently, the number of active sites in line with most observations. 7,11,19,21,22 However, the degradation of the dye decreased with the increase of the catalyst load to 20.0 $\mathrm{mg}$ (in $200 \mathrm{ml}$ dye solution). In a photocatalytic reaction, the catalyst is known to have an 
optimum amount, and an amount more than this causes unfavourable light scattering and reduces light penetration into the solution. Thus LDH loading of $15.0 \mathrm{mg}$ per $200 \mathrm{ml}$ of the dye solution $\left(1 \times 10^{-5} \mathrm{M}\right)$ could be considered as the optimum for RhB degradation.

3.7.2. Effects of $\mathbf{p H}$.-The photoassisted degradation of $\mathrm{RhB}$ was carried out with 15.0 $\mathrm{mg} \mathrm{LDH}$ per $200 \mathrm{ml}$ of the dye solution $\left(1 \times 10^{-5} \mathrm{M}\right)$ in the $\mathrm{pH}$ range of 4.0 to 11.0. The plots of $C / C_{o}$ vs. irradiation time are presented in Fig. 9(B). It is seen that in acidic medium, the degradation is low, because at low $\mathrm{pH}, \mathrm{H}^{+}$ions compete with $\mathrm{RhB}$ cations for the available adsorption sites on the LDH surface. The LDH surface is positive below its isoelectric point of 7.16 resulting in an electrostatic repulsion of $\mathrm{RhB}$ cations at lower $\mathrm{pH}$, and consequently less adsorption of the dye cations on LDH surface and less degradation. In alkaline medium, the LDH surface becomes increasingly negative and degradation increases. 5,16,22 The maximum degradation effciency of $\sim 99.8 \%$ was observed at $\mathrm{pH} 11$.

3.7.3. Effects of initial dye concentration.-The photoassisted decolorization of Rhodamine B was carried out at the optimum $\mathrm{pH}(=11)$ with $15.0 \mathrm{mg}$ of $\mathrm{LDH} / 200 \mathrm{ml}$ of dye solution at $30^{\circ} \mathrm{C}$ [Fig. 9(C)] with three different dye concentrations of $1 \times 10^{-3}, 1 \times 10^{-4}$ and $1 \times 10^{-5} \mathrm{M}$. Around $99.8 \%$ degradation of $\mathrm{RhB}$ was achieved with $1 \times 10^{-5} \mathrm{M}$ solution of the dye. Above this concentration, the degradation decreased to $77 \%$ and $54 \%$ respectively for $1 \times 10^{-4} \mathrm{M}$ and $1 \times 10^{-3} \mathrm{M}$ dye solution. With increasing dye concentration, more dye molecules are likely to be adsorbed on the LDH surface and accumulation of the adsorbed dye causes an inhibitive effect on their photocatalytic degradation due to lack of their direct contact between the photogenerated holes, hydroxyl radicals, superoxide radicals (generated on the LDH during photocatalysis). Moreover, high dye concentration most likely acts as an inner filter which shunts the photons away from the LDH surface. The dye molecules after adsorption blocks the active surface sites on the LDH and hence the degradation efficiency decreases. $5,16,19,22$

3.7.4. Characterizations of active species.-Photocatalysis by a semiconductor material is driven primarily by active species like $\bullet \mathrm{OH}, \mathrm{h}^{+}$and $\mathrm{O}_{2}{ }^{-}{ }^{-}$species. The presence of $\bullet \mathrm{OH}$ radicals during the photoassisted degradation was measured using terephthalic acid (TA) fluorescence probe. In this case, $1 \times 10^{-5} \mathrm{M}$ TA in $4 \times 10^{-5} \mathrm{M}$ aqueous $\mathrm{NaOH}$ replaced the dye solution in the photocatalytic reactor. TA undergoes reaction with $\bullet \mathrm{OH}$, generating a highly fluorescent compound 2-hydroxyterephthalic acid; which shows photoluminescence emission at $426 \mathrm{~nm}$, when subjected to excitation at $315 \mathrm{~nm}$ wavelength [Fig. 9(D)]. However, no photoluminescence signal was observed when the experiment was carried out without visible light irradiation indicating the generation of $\bullet \mathrm{OH}$ radicals by the $\mathrm{LDH}$ under visible light. ${ }^{23}$ The surface $-\mathrm{OH}$ groups present on the LDH are most likely to transform themselves to $\bullet \mathrm{OH}$ radicals that control the photocatalysis process. The presence of $\bullet \mathrm{OH}$ radicals could be further confirmed by external addition of $n$-butanol (quencher of $\bullet \mathrm{OH}$ ) prior to the photocatalysis reaction under the same set of experimental conditions. ${ }^{16}$ It is seen that the photodegradation of the dye was suppressed to a considerable extent, indicating that $\bullet \mathrm{OH}$ was one of the active species that controlled the photodegradation of the dye [Fig. 9(E)]. EPR spectroscopy was used to study the presence of superoxide $\left(\mathrm{O}_{2}{ }^{-}\right)$species generated on the LDH surface during photo-catalysis. The EPR analysis was done using a 
Bruker EMX X-band spectrometer with $100 \mathrm{kHz}$ field modulation. The frequency calibration was done with a frequency counter microwave bridge (ER 041 XG-D) with DPPH being used as the field marker ( $g=2.0036)$. A HPA 400/30 S lamp (400 W, Philips) was used as irradiating light source for EPR analysis. The magnitude of magnetic field of the radicals was determined using the formula $h v=g \beta H$. Prior to EPR analysis, the LDH was subjected to thermal treatment at $473 \mathrm{~K}$ in vacuum followed by exposure to an oxygen environment at room temperature. The EPR spectrum of the LDH [Fig. 9(F)] analysed in $\mathrm{O}_{2}$ environment under visible light $(\lambda>400 \mathrm{~nm})$ showed signals at $\mathrm{g}_{\mathrm{x}}=2.0037, \mathrm{~g}_{\mathrm{y}}=2.011$ and $\mathrm{g}_{\mathrm{z}}=2.003$, which coincide with the $g$-tensor of the $\mathrm{O}_{2}{ }^{{ }^{-}}$species. This indicates that $\mathrm{O}_{2}{ }^{\cdot-}$ species lead to the generation of visible light sensitive redox centers, which are primarily responsible for the dye degradation.

Apart from EPR analysis, an indirect radical trapping experiment was also performed with the test dye by external addition of benzoquinone (BQ; $\mathrm{O}_{2}{ }^{-}$scavenger) prior to visible light irradiation, under the same set of photocatalytic conditions. A decrease in degradation of Rhodamine B [Fig. 9(E)] confirmed that $\mathrm{O}_{2}{ }^{--}$was one of the most active species driving the photocatalysis. ${ }^{16,23}$

The photogenerated holes in a semiconductor material, also play a major role in the degradation of the dye. ${ }^{24}$ The generation of holes during the visible light assisted photocatalysis was investigated by external addition of the quencher, EDTA-2Na to the experimental solution, prior to visible light irradiation. The suppression of degradation [Fig. 9(E)], suggested that the photogenerated holes have played an important part in the degradation of the dye. ${ }^{16}$ Thus, the photocatalysis by the LDH is assisted by the active species, $\mathrm{O}_{2}^{\cdot-}, \mathrm{h}^{+}$and ${ }^{\circ} \mathrm{OH}$ radicals.

3.7.5. Kinetics.-The kinetics of photoassisted degradation was evaluated under optimum conditions ( $\mathrm{pH} 11, \mathrm{LDH} 15.0 \mathrm{mg}$; $\mathrm{RhB} 1 \times 10^{-5} \mathrm{M}$, dye solution $200 \mathrm{ml}$ ) to understand the process catalysed by $\mathrm{Mn} / \mathrm{Co} / \mathrm{Ti} \mathrm{LDH}$ under visible light. The plot of $\ln \left(\mathrm{C}_{\mathrm{o}} / \mathrm{C}\right)$ vs. irradiation time [S7; Fig. S6 (ESI $\dagger)$ ] is close to linearity, indicating that $\mathrm{RhB}$ degradation follows pseudo first order kinetic model represented by eqn (5)

$$
\ln \left(\frac{C_{\mathrm{o}}}{C}\right)=k_{\mathrm{app}} t
$$

where $C_{\mathrm{o}}$ is the initial concentration of the dye and $C$ is the concentration at time $t$. The apparent rate constant $k_{\text {app }}$ was calculated to be $0.041 \mathrm{~min}^{-1}$ for RhB. The calculated half life was found to be $16.91 \mathrm{~min}$ for $\mathrm{RhB}$ using the eqn (6)-

$$
t_{1 / 2}=\frac{\ln 2}{k_{\text {app }}}
$$

The pseudo first order kinetic model suggests that the photocatalytic reaction rate is primarily dependent on light intensity and the absorption efficiency of the LDH. 
3.7.6. Comparative performance of the LDH with commercial catalysts.-A comparative analysis of $\mathrm{RhB}$ degradation by the synthesized $\mathrm{LDH}$ and the commercial catalysts, $\mathrm{MnO}_{2}, \mathrm{TiO}_{2}, \mathrm{CoO}$ and Degussa $\mathrm{P} 25$ under visible light showed a higher photocatalytic efficiency of the LDH. Control experiments (blank and dark reactions) with the LDH indicated negligible performance and therefore, the dye degradation is controlled by the intensity of visible light only. The results are illustrated in Table 3 and Fig. 10, which suggest a superior performance of the LDH over the commercial catalysts as a photocatalyst for RhB degradation.

3.7.7. Recyclability and photostability of the LDH.-The reusability of the LDH photocatalyst is a parameter of vital importance from the industrial perspective. After each photocatalytic cycle, the LDH was recovered from the reaction mixture by centrifugation, washed several times with water and dried. The recyclability of the LDH was tested upto fifth cycle of RhB degradation. The degradation of RhB in the first cycle was $99.8 \%$, which decreased by some extents in the next cycles reaching $~ 87 \%$ after the fifth cycle [S8; Fig. S7 (ESI $\dagger)]$. This illustrates that the LDH maintains good photoactivity for degradation of $\mathrm{RhB}$ even after the fifth cycle. The LDH is a heterogeneous photocatalyst that can be easily recycled by a simple filtration because of its higher density. The decrease in the photocatalytic efficiency after its reuse is most likely to be caused by the reduction of a number of active sites available on the LDH surface after each cycle of photodegradation. However, it was observed that even after five degradation cycles, the LDH did not exhibit much loss of activity confirming that the catalyst was not photo-corroded during the reactions. The stability of the new photocatalyst is important with respects to its application and it is to be noted that the doped Ti-photocatalysts sometimes have this problem. ${ }^{37,38}$ Moreover, the linear nature of each curve suggests that the degradation kinetics follows pseudo-first order model, in all the degradation cycles.

A comparative FT-IR analysis of the pure LDH and that recovered after the fifth cycle of RhB degradation [S9; Fig. S8 (ESI $\dagger$ )] showed that the material also possess very good photo-stability. The IR bands remained at nearly at the same positions with some minor changes being observed in the fingerprint region of the $\mathrm{LDH}$.

3.7.8. FT-IR analysis of the end product of photocatalysis.-The occurrence of adsorption or degradation in the decolorization of aqueous $\mathrm{RhB}$ is determined by comparative FT-IR analysis of the pure dye with that of the centrifugate of the dark reaction, blank reaction and the colourless end product of photocatalysis brought about by $2: 1: 1$ $\mathrm{Mn} / \mathrm{Co} / \mathrm{Ti} \mathrm{LDH}$ under visible light irradiation [S10; Fig. S9 (ESI $\dagger$ )]. It is observed that all the characteristic bands appear at their respective positions for the control experiments (blank and dark reactions) with some broad peaks in the fingerprint region. The bands appeared to be broad indicating that adsorption dominated the control experiments. However, the FT-IR spectra of the colourless end products of the reaction showed the absence of most of the characteristic bands of $\mathrm{RhB}$, instead some new bands appeared at different positions, signifying that the complex dye was degraded to simple molecules.

3.7.9. Carbon mass balance.-Carbon balancing is a unique technique for assessing the accuracy of performance during the measurements. Good carbon mass balance closure 
reflects the consistency of the internal data and provides a reasonable confidence in the accuracy of the underlying data. If carbon mass balance approaches $100 \%$ or very similar values, the stoichiometric accuracy of the process could be considered as very reliable.

In order to investigate the carbon mass balance, small ports were configured within the reactor top for input of the reactants and an exit port for output of the effluent. Influent and effluent were typically collected in $5 \mathrm{~min}$ intervals. These were quantified within the photocatalytic system with the help of (a) GC-FID (b) ion chromatograph and (c) TOC analyser. The inlet and outlet components were monitored throughout the experiments to determine the overall mass balance. The concentrations were converted to cumulative moles of carbon. Inlet carbon content was compared to carbon accumulated in the reactor and carbon existing in the effluent to find out the overall mass balance for the system. Chloride concentrations were below the accurate range of the ion chromatograph and negligible in comparison to the other constituents, and were not reported. Fig. 11 presents the overall carbon mass balance of the system.

It is seen that the inlet and outlet moles of carbon were nearly equal in the initial stages of experimentation; however the accumulated and effluent carbon fall to approximately $92 \%$ of the estimated inlet carbon content. The HPLC/MS used for the quantification of the dye does not indicate any presence of the parent dye content in the effluent. The carbon mass balance results confirm mineralization of the dye through a relatively rapid pathway.

\subsubsection{Morphology and elemental composition of the spent LDH.-The TEM}

analysis of the recovered catalyst revealed the existence of hexagonal nanoparticles stacked one over the other like that observed for the pure LDH. No drastic change in the morphology was observed for the LDH even after the fifth cycle (Fig. 12). The elemental composition of the LDH recovered after the fifth cycle of degradation was investigated by EDX measurements (Table 4). Minor changes in the weight and atomic percentages in the LDH could be observed, but $\mathrm{Mn} / \mathrm{Co} / \mathrm{Ti}$ ratio remained unaltered $(=2.06: 1.04: 1.00)$ even after the fifth cycle of degradation. This reflected stability of the LDH under the chemical environments of photodegradation even after the fifth cycle.

3.7.11. Mineralization study.-The final products from the photocatalytic conversion of the dye should be some intermediates, $\mathrm{CO}_{2}$ and $\mathrm{H}_{2} \mathrm{O}$. The intermediates are most likely to be aromatic compounds accounting for the remaining TOC of the reaction mixture. Various aromatic degradation products have been reported and the complete mineralization of the dye may not have been achieved. The mineralization was monitored by continuously following the TOC (total organic carbon) of the reaction mixture. ${ }^{36}$ The percentage reductions in TOC with respect to time for visible light irradiated dye solution, and that for the dye solution irradiated with visible light as well as treated with $\mathrm{H}_{2} \mathrm{O}_{2}$ (S11; Fig. S10; $\mathrm{ESI} \dagger$ ) showed that TOC was reduced by $92 \%$ of the original when the degradation was carried out under visible light alone. Almost 100\% reduction could be achieved within 60 min, whereas $92 \%$ reduction in TOC was observed after $60 \mathrm{~min}$ through photocatalysis by the LDH. Thus it could be inferred that the TOC removal rate is less in the photocatalytic degradation compared to that of $\mathrm{H}_{2} \mathrm{O}_{2}$ assisted decolorization under similar experimental 
conditions. This is obviously due to the formation of more stable intermediates in the photocatalytic process as identified using GC-MS and ex situ ${ }^{1} \mathrm{H}-\mathrm{NMR}$ studies.

3.7.12. Mechanistic pathways of photodegradation.-On the basis of the results obtained from XPS, PL, EIS, DRS, TA fluorescence probe, EPR and indirect radical and hole trapping experiments, the mechanistic pathways of the degradation of RhB by $\mathrm{Mn} / \mathrm{Co} / \mathrm{Ti} \mathrm{LDH}$ could be suggested. In accordance with the band theory, $\mathrm{O} 1 \mathrm{~s}$ orbital could be considered as the valence band and Mn 2p, Co 2p and Ti 2p energy levels as the conduction band. The photocatalytic degradation is likely to proceed in two different pathways, through generation of reactive species like ${ }^{\circ} \mathrm{OH}$ and $\mathrm{O}_{2}{ }^{--}$in presence of visible light.

Pathway 1: electron-hole $\left(\mathbf{e}-\mathbf{h}^{+}\right)$hopping conduction mechanism.: A series of reactions is likely to take place in the sequential pattern (eqn (7) to (19)) upon visible light exposure. The electrons are first excited from the valence band to the conduction band, thereby leaving holes in the valence band. The absorption of photons initiates electronic excitation with energy equal to or greater than the band gap energy of the semiconductor $\mathrm{Mn} / \mathrm{Co} / \mathrm{Ti} \mathrm{LDH}$ nanomaterial. Thus, $2: 1: 1 \mathrm{Mn} / \mathrm{Co} / \mathrm{Ti} \mathrm{LDH}$ generates $\mathrm{e}^{-}-\mathrm{h}^{+}$pairs (eqn (7)) upon visible light exposure, reducing $\mathrm{Mn}^{3+}, \mathrm{Co}^{3+}$ and $\mathrm{Ti}^{4+}$ species on the $\mathrm{LDH}$ surface (eqn (8)-(10)). A strong redox reaction ensues (eqn (11)) initiated by the photo-generated electrons with intense lattice vibrations on the LDH surface. $\mathrm{h}^{+}$hops within the LDH surface and reacts with $\mathrm{H}_{2} \mathrm{O}$ and $\mathrm{OH}^{-}$ions yielding $\mathrm{OHc}$ radicals (eqn (12) and (13)). The photogenerated electrons generated is likely to react with adsorbed oxygen species producing superoxide $\left(\mathrm{O}_{2}{ }^{--}\right)$radicals (eqn (14)). Superoxide $\left(\mathrm{O}_{2}{ }^{\cdot-}\right)$ species undergo further transformation with the adsorbed $\mathrm{H}^{+}$present on the $\mathrm{LDH}$ surface to produce peroxide radicals $\left({ }^{\circ} \mathrm{OOH}\right)($ eqn (15)), further yielding $\mathrm{H}_{2} \mathrm{O}_{2}$ (eqn (16)) as well as hydroxyl radicals ( ${ }^{\circ} \mathrm{OH}$ ) (eqn (17)). Hydroxyl $(\mathrm{cOH})$ and superoxide $\left(\mathrm{O}_{2}{ }^{\circ-}\right)$ radicals are highly reactive species that mineralize the dye to simple molecules (eqn (18)). This follows the recombination between $\mathrm{h}^{+}$and $\mathrm{e}^{-}$ with dissipation of energy and reduction of quantum efficiency (eqn (19)). The pathway involves the sequence of reactions as follows:

$$
\begin{gathered}
\mathrm{Mn} / \mathrm{Co} / \mathrm{Ti} \mathrm{LDH}+h v \rightarrow \mathrm{Mn} / \mathrm{Co} / \mathrm{Ti} \mathrm{LDH},\left(\mathrm{e}^{-}+\mathrm{h}^{+}\right) \\
\mathrm{Mn}^{3+}+\mathrm{e}^{-} \rightarrow \mathrm{Mn}^{2+},(\text { electron trap) } \\
\mathrm{Co}^{3+}+\mathrm{e}^{-} \rightarrow \mathrm{Co}^{2+},(\text { electron trap) } \\
\mathrm{Ti}^{4+}+\mathrm{e}^{-} \rightarrow \mathrm{Ti}^{3+},(\text { electron trap) }
\end{gathered}
$$




$$
\begin{aligned}
& \mathrm{Mn}^{3+}+\mathrm{Co}^{3+}+\mathrm{Ti}^{4+}+3 \mathrm{e}^{-}+h v \leftrightarrow \mathrm{Mn}^{2+}+\mathrm{Co}^{2+}+\mathrm{Ti}^{3+}, \text { (migration) } \\
& \mathrm{h}^{+}+\mathrm{H}_{2} \mathrm{O} \rightarrow \mathrm{HO}^{\cdot}+\mathrm{H}^{+} \\
& \mathrm{h}^{+}+\mathrm{OH}^{-} \rightarrow \mathrm{HO}^{\circ} \\
& \mathrm{O}_{2}+\mathrm{e}^{-} \rightarrow \mathrm{O}_{2}^{\cdot-} \\
& \mathrm{O}_{2}^{\cdot-}+\mathrm{H}^{+} \rightarrow \mathrm{HOO}^{\cdot} \\
& \mathrm{HOO}^{\circ}+\mathrm{HOO}^{\circ} \rightarrow \mathrm{H}_{2} \mathrm{O}_{2}+\mathrm{O}_{2} \\
& \mathrm{H}_{2} \mathrm{O}_{2}+\mathrm{O}_{2}^{\cdot-} \rightarrow \mathrm{HO}^{\cdot}+\mathrm{OH}^{-}+\mathrm{O}_{2} \\
& \mathrm{RhB} \text { dye }+\mathrm{O}_{2}^{\cdot-}+\mathrm{HO}^{\cdot} \rightarrow \text { degradation products } \\
& \mathrm{h}^{+}+\mathrm{e}^{-} \rightarrow \text { energy }
\end{aligned}
$$

Pathway 2: by photosensitization of RhB.: This pathway proceeds through the photosensitization of $\mathrm{RhB}$ under visible light. The dye molecule acts as a photosensitizer to yield its excited state (eqn (20)), which is likely to transform further to dye radical with the release of electron (eqn (21)). The dye radical forms the most likely intermediate products like $\mathrm{CO}_{2}$ and $\mathrm{H}_{2} \mathrm{O}$ (eqn (22)). The electrons generated are injected into the conduction band of the semiconductor $\mathrm{LDH}$ (eqn (23)) where they are scavenged by $\mathrm{O}_{2}$ forming $\mathrm{O}_{2}{ }^{--}$species (eqn (24)), also yielding $\mathrm{H}_{2} \mathrm{O}_{2}$ (eqn (25)). $\mathrm{H}_{2} \mathrm{O}_{2}$ subsequently generates ${ }^{\bullet} \mathrm{OH}$ radicals (eqn (26)), which further degrades the complex dye to simple molecules (eqn (27)). The sequential series of reactions are as follows: 


\author{
$\mathrm{RhB}$ dye $+h v \rightarrow$ dye $*$ \\ RhBdye $^{*} \rightarrow$ RhBdye ${ }^{+}+\mathrm{e}^{-}$ \\ RhBdye ${ }^{+} \rightarrow \mathrm{CO}_{2}+\mathrm{H}_{2} \mathrm{O}$ \\ $\mathrm{Mn} / \mathrm{Co} / \mathrm{Ti} \mathrm{LDH}+\mathrm{e}^{-} \rightarrow \mathrm{Mn} / \mathrm{Co} / \mathrm{Ti}_{\mathrm{LDH}}{ }^{+}$ \\ $\mathrm{Mn} / \mathrm{Co} / \mathrm{Ti} \mathrm{LDH}^{\cdot+}+\mathrm{O}_{2} \rightarrow \mathrm{Mn} / \mathrm{Co} / \mathrm{Ti} \mathrm{LDH}+\mathrm{O}_{2} \cdot-$ \\ $\mathrm{O}_{2} \cdot{ }^{-}+2 \mathrm{H}_{2} \mathrm{O}+\mathrm{e}^{-} \rightarrow 2 \mathrm{H}_{2} \mathrm{O}_{2}$ \\ $\mathrm{H}_{2} \mathrm{O}_{2}+\mathrm{e}^{-} \rightarrow \mathrm{OH}+\mathrm{OH}^{-}$ \\ $\mathrm{OH}+\mathrm{RhBdye} \rightarrow$ degradation products

The mechanistic pathways clearly demonstrate the involvement of holes $\left(\mathrm{h}^{+}\right)$, hydroxyl $(\mathrm{cOH})$ and superoxide $\left(\mathrm{O}_{2}{ }^{--}\right)$species as highly reactive agents for $\mathrm{Mn} / \mathrm{Co} / \mathrm{Ti} \mathrm{LDH}$ mediated aqueous phase photocatalytic degradation of the cationic Rhodamine B (RhB). The XPS and PL measurements suggest the presence of multiple valencies of Mn, Co and Ti accompanied by oxygen vacancies within the $\mathrm{LDH}$. The presence of defects and $\mathrm{O}_{2} / \mathrm{O}_{2}{ }^{--}$transformation in the LDH is confirmed by EIS and EPR analyses. The presence of hydroxyl radical ( $\left.{ }^{\circ} \mathrm{OH}\right)$ is confirmed by terephthalic acid (TA) fluorescence probe. The presence of holes $\left(\mathrm{h}^{+}\right)$, hydroxyl radical $\left({ }^{\circ} \mathrm{OH}\right)$ and superoxide $\left(\mathrm{O}_{2}{ }^{-}\right)$species are confirmed by indirect radical and hole trapping experiments by addition of appropriate quenchers at the beginning of the photocatalytic degradation of RhB. Depending on these observations, the mechanistic pathways have been presented in the form of $\mathrm{e}^{-}-\mathrm{h}^{+}$hopping conduction model and by photosensitization of the dye. The schematic of photodegradation pathway is illustrated in Fig. 13. 


\subsubsection{Identification of end products and elucidation of pathways of photodegradation.-Ex situ ${ }^{1} \mathrm{H}-\mathrm{NMR}$ and GC-MS analysis of the colourless end products of $\mathrm{RhB}$ degradation helped to elucidate the mechanistic pathways of photodegradation.}

Ex situ ${ }^{1} \mathbf{H}$-NMR analysis.: The elemental $\mathrm{H}$-atoms present in the structure of the cationic dye, RhB (Fig. 14) are shown in the ${ }^{1}$ H-NMR spectrum of Rhodamine B [Fig. 15(a)] and the colourless photodegraded products [Fig. 15(b)]. The pure dye shows signals of aromatic $\mathrm{H}-$ atoms $-\mathrm{H}_{\mathrm{c}}, \mathrm{H}_{\mathrm{d}}, \mathrm{H}_{\mathrm{e}}, \mathrm{H}_{\mathrm{f}}, \mathrm{H}_{\mathrm{g}}, \mathrm{H}_{\mathrm{h}}$, and $\mathrm{H}_{\mathrm{i}}(\mathrm{c}, \mathrm{d}, \mathrm{e}, \mathrm{f}, \mathrm{g}$, h and $\mathrm{i}$ are the respective positions of elemental $\mathrm{H}$-atoms present in the structure of the cationic $\mathrm{RhB}$ ) consistent with the previous literature. ${ }^{25-27}$ These NMR signals are observed at $\delta$ values of $6.05,6.13,6.78,7.35,7.58$, 7.29 and $7.93 \mathrm{ppm}$ respectively. Moreover, $\mathrm{H}_{\mathrm{a}}$ represents the aliphatic hydrogen in $\mathrm{N}$-diethyl amine group for $-\mathrm{CH}_{3}$ with chemical shift being observed at $\sim \delta(1.14)$ ppm and $\mathrm{H}_{\mathrm{b}}$ representing the methylene $\left(>\mathrm{CH}_{2}\right)$ group of $\mathrm{N}, \mathrm{N}$-diethyl amine which was observed at $\sim \delta$ (3.39) ppm chemical shift. Moreover, a number of ${ }^{1} \mathrm{H}-\mathrm{NMR}$ signals of the pure dye appeared at $\mathrm{d}$ values of $0.92,1.45,4.56,4.95,5.75$ and $11.05 \mathrm{ppm}$, which could be attributed to that of $\mathrm{H}_{\mathrm{a}}{ }_{\mathrm{a}}, \mathrm{H}{ }_{\mathrm{b}}, \mathrm{H}_{\mathrm{c}}{ }_{\mathrm{c}}, \mathrm{H}_{\mathrm{d}}, \mathrm{H}_{\mathrm{e}}{ }_{\mathrm{e}}$ and $-\mathrm{OH}$ groups of the carboxylic acid. The occurrence of these ${ }^{1} \mathrm{H}-$ NMR signals corroborates with the previous literature. ${ }^{25-27}$

However, several interesting features emerge when ${ }^{1} \mathrm{H}-\mathrm{NMR}$ spectra of the colourless end products were compared with those of pristine Rhodamine B. Thus, it revealed the disappearance of peaks at chemical shift ( $\delta$ ) values of 6.0-7.8 ppm, preferably dominated by protons associated with aromatic moieties. The disappearance reflects the absence of an aromatic moiety that existed in pure Rhodamine $\mathrm{B}$, and therefore, complete degradation of the dye. It also suggests preferable paths through which photocatalytic cleavage of Rhodamine B could occur. ${ }^{25-27}$ Simultaneously, several new peaks are observed in the ${ }^{1} \mathrm{H}$ NMR spectra of the decolorized end products having chemical shift of $(0-4.0) \mathrm{ppm}$.

The disappearance of $\mathrm{H}_{\mathrm{f}}$ to $\mathrm{H}_{\mathrm{i}}$ and the presence of only one aromatic proton post irradiation shows the possibility of occurrence of the cleavage pathway 1 (Fig. 16) leading to the formation of benzoic acid. The formation of the benzoic acid as the visible photodegradation product of $\mathrm{RhB}$ corroborates with the GC-MS data of the dye metabolite. ${ }^{25-27}$ Moreover, the loss of $\mathrm{H}_{\mathrm{c}}$ and $\mathrm{H}_{\mathrm{d}}{ }^{1} \mathrm{H}$-NMR signals after the photocatalysis indicates the occurrence of cleavage pathway 2 (Fig. 16). This may lead to the formation of the amides and subsequent amines and diols. However, there is a possibility of simultaneous cleavage through $(2+2)$ [Fig. 16], which would lead to the formation of diols, primary acids, certain amines and benzoic acid. These products can be cleaved further, forming smaller molecules like acetic acid and formic acid respectively. The product formation is further supplemented by the appearance of a number of new peaks during the photodegradation of $\mathrm{RhB}$. The NMR peak at $\mathrm{d}(0.58) \mathrm{ppm}$ is a sharp high intensity signal, generally due to the presence of primary alcohol or acetyl group. A small broad peak at $\mathrm{d}(0.68)$ ppm suggests the formation of $-\mathrm{H}$ of an amide or probably an alkyl $-\mathrm{CH}_{3}$ group. ${ }^{28-35}$ The occurrence of two sharp peaks at $\delta=$ $0.78 \mathrm{ppm}$ indicates $-\mathrm{H}$ of a $-\mathrm{CH}_{3}$ group and at $\mathrm{d}(1-1.2)$ ppm shows a doublet $-\mathrm{H}$, associated with that of a primary alcohol. The existence of a new peak at $\delta(2-2.2) \mathrm{ppm}$ indicates the presence of acetic acid. The formation of secondary amine could be represented by the peak at $\delta(3.15) \mathrm{ppm}$. Moreover, several peaks between $\delta(3.2-3.8) \mathrm{ppm}$, 
represents different alcohols and phenolic groups. Again, the strong peak at $\delta(3.8-4.0) \mathrm{ppm}$ suggests the existence of an amide or ester. The peaks at $\delta(1-1.2) \mathrm{ppm}$ and $\delta(3.3-3.4) \mathrm{ppm}$ even after complete decolorization of the dye after $60 \mathrm{~min}$ of photodegradation suggests the absence of the de-ethylation pathway for photo dissociation and degradation of $\mathrm{RhB}$ by the LDH.

GC-MS analysis of the decolorized dye metabolites.: The analysis of the decolorized final products of photodegradation was further done by GC-MS to crosscheck the information obtained from ${ }^{1} \mathrm{H}$-NMR spectra and hence was used to elucidate the pathway of dye decolorization. The GC-MS results supported the asymmetric cleavage of Rhodamine B through different pathways and the breakdown products were identified on the basis of the $\mathrm{m} / \mathrm{z}$ values of the components (S12; Fig. S11; ESI $\dagger$ ). The degradation products were collected after the decolorization efficiency reached the maximum (reaction time $60 \mathrm{~min}$ ), visible light irradiation, $15.0 \mathrm{mg}$ of $\mathrm{Mn} / \mathrm{Co} / \mathrm{Ti} \mathrm{LDH}$ in $200 \mathrm{ml}$ dye solution of concentration $1 \times 10^{-5} \mathrm{M}$. The reaction end products were extracted with dichloromethane and $1 \mathrm{ml}$ of the same was injected into the GC-MS, MS analysis being done in positive-ion mode. It is evident that the photocatalytic degradation generates ${ }^{\circ} \mathrm{OH}$ radicals and $\mathrm{O}_{2}{ }^{\circ-}$ species that catalyse the aqueous phase degradation of $\mathrm{RhB}$.

Eight peaks could be identified in the mass spectrum of the degradation products of Rhodamine B [S12; Fig. S11; ESI $\dagger$ ]. The analysis of mass spectra suggests that the mechanistic pathway of degradation proceeds with the asymmetric cleavage of the dye (Fig. 16), which is likely to undergo transformations in three possible pathways. In pathway 1 , the dye breaks down to benzoic acid $(\mathrm{m} / \mathrm{z}=122.02)$. The pathway 2 is most likely to proceed through the formation of breakdown products like $N, N$-diethylacetamide $(m / z=115.08)$, [4(2-carboxyphenyl)-2-methyl chromen-7-ylidene]-diethylammonium ion $(\mathrm{m} / \mathrm{z}=336.18)$ and diethyl-(2-methylchromen-7-ylidene)-ammonium ion $(\mathrm{m} / \mathrm{z}=216.13)$. The third pathway ( 2 +2 ), is likely to proceed through the formation of 2-(6-methyl-3,4-dihydro-2H-pyran-4-yl)benzoic acid $(\mathrm{m} / \mathrm{z}=218.07)$, which further breaks down to form butane-2,3-diol $(\mathrm{m} / \mathrm{z}=$ 88.07), acetic acid $(\mathrm{m} / \mathrm{z}=60.05)$, formic acid $(\mathrm{m} / \mathrm{z}=45.97)$ and benzoic acid $(\mathrm{m} / \mathrm{z}=$ 122.02). The existence of these breakdown products in the mass spectra supports the asymmetric cleavage of the dye, suggesting the remarkable ability of the LDH to bring about photodegradation of the dye.

\section{Conclusions}

$2: 1: 1 \mathrm{Mn} / \mathrm{Co} / \mathrm{Ti} \mathrm{LDH}$ has displayed a remarkable photo-catalytic degradation efficiency for Rhodamine $\mathrm{B}$ in aqueous solution, much higher than that of commercial catalysts, $\mathrm{MnO}_{2}, \mathrm{TiO}_{2}, \mathrm{CoO}$ and Degussa P25. This could be attributed to the presence of a narrow band gap, high surface area, layered mesoporous structure with defects and oxygen vacancies in the LDH. The XPS provided information about the presence of different surface states with oxygen vacancies within the layered framework. It is found that adsorption dominated the control experiments whereas degradation marked the end of the photochemical processes with the LDH. A high catalytic efficiency of the synthesized LDH is retained even after the fifth cycle and its FT-IR analysis before and at the end of the fifth cycle showed remarkable stability. The degradation kinetics followed pseudo first order 
model for the test dye. The photo-catalysis proceeds through electron-hole hopping and by dyesensitized mechanistic pathways. Information obtained through ex situ ${ }^{1} \mathrm{H}-\mathrm{NMR}$ and GC-MS analyses have been helpful in elucidation of the reaction pathways through the identified breakdown products of the dyes. The carbon mass balance analysis showed that mineralization of the dye could be achieved through a relatively rapid pathway. There was a $92 \%$ reduction of TOC in the photocatalytic process, which was slightly less than the almost $100 \%$ decolorization achieved with chemical oxidation under the similar experimental conditions. This work has provided new insights into photocatalysis by the synthesized LDH consistent with a cost-effective and highly efficient degradation of dyes for large scale environmental wastewater treatment.

\section{Supplementary Material}

Refer to Web version on PubMed Central for supplementary material.

\section{Acknowledgements}

The authors are thankful to Professor Sanjeev K. Srivastava, Department of Physics and Meteorology, Indian Institute of Technology, Kharagpur (India) for helping with the XPS analysis of the LDH. Moreover, PRC is grateful to University Grants Commission (UGC), New Delhi, India for providing financial assistance under UGCBSR Fellowship Scheme. The authors are thankful to the reviewers for suggesting certain valuable inputs to the manuscript.

\section{References}

1. Liu B , Nakata K, Sakai M, Saito H, Ochiai T, Murakami T, Takagi K and Fujishima A, Catal. Sci. Technol, 2012, 2, 1933.

2. Fujishima A and Honda K, Nature, 1972, 238, 37.12635268

3. Xiang Q and Yu J , J. Phys. Chem. Lett, 2013, 4, 753.26281930

4. Silva NU, Nunes TG, Saraiv MS, Shalamzari MS, Vaz PD, Monteiro OC and Nunes CD , Appl. Catal., B, 2012, 113-114, 180.

5. Roy Chowdhury P and Bhattacharyya KG , Dalton Trans, 2015, 44, 6809.25763803

6. Zhang N, Yang MQ, Liu S, Sun Y and Xu YJ , Chem. Rev, 2015, 115, 10307.26395240

7. Zhao Y, Li B , Wang Q, Gao W, Wang CJ , Wei M, Evans DG, Duan X and O'Hare D, Chem. Sci, 2014, 5, 951.

8. He J , Wei M , Li B , Kang Y , Evans DG and Duan X, Struct. Bonding, 2006, 119, 89.

9. Wang Q and O'Hare D, Chem. Rev, 2012, 112, 4124.22452296

10. Štengl V and Bakardjieva S, J. Phys. Chem. C, 2010, 114, 19308.

11. Li YX, Chen J and Wang L, J. Photochem. Photobiol., A, 2004, 163, 517.

12. Song $\mathrm{F}$ and $\mathrm{Hu} \mathrm{X}$, Nat. Commun, 2014, 5, 1.

13. Shao M , Han J , Wei M , Evans DG and Duan X, Chem. Eng. J, 2011, 168, 519.

14. Selvam T , Inayat A and Schweiger W , Dalton Trans, 2014, 43, 10365.24841986

15. Biesinger MC, Payne BP, Grosvenor AP, Lau LWM, Gerson AR and Smart RSC, Appl. Surf. Sci, 2011, 257, 2717.

16. Roy Chowdhury P and Bhattacharyya KG, RSC Adv, 2015, 5, 92189.

17. Bard AJ , Fan FRF, Gioda AS , Nagasubramanian G and White HS , Faraday Discuss. Chem. Soc, 1980, 70, 19.

18. Choudhury B , Dey M and Choudhury A, Appl. Nanosci, 2014, 4, 499.

19. Mahapatra L and Parida KM , J. Mater. Chem. A, 2016, 4, 10744.

20. Zhang N , Zhang Y and Xu YJ , Nanoscale, 2012, 4, 5792.22907128 
21. Comparelli R , Fanizza E, Curri ML, Cozzoli PD , Mascolo G and Agostiano A, Appl. Catal., B, 2005, 60, 1 .

22. Zhao YF, Wei M, Lu J, Wang ZL and Duan X, ACS Nano, 2009, 3, 4009.19928881

23. Mohapatra L and Parida KM , Phys. Chem. Chem. Phys, 2014, 16, 16985.25005613

24. Sharma M , Ojha K, Ganguly A and Ganguli AK , New J. Chem, 2015, 39, 9242.

25. Li J , Ma W, Lei P and Zhao J , J. Environ. Sci, 2007, 19, 892.

26. Liu G and Zhao J , New J. Chem, 2000, 24, 411.

27. Wu T, Liu G, Zhao J , Hidaka H and Serpone N , J. Phys. Chem. B, 1998, 102, 5845.

28. Yu S , Yang H , He C, Sun C and Ju Y , J. Phys. Chem. A, 2009, 102, 10024.

29. Silverstein RM and Webster FX, Spectrometric Identification of Organic Compounds, Wiley Student Edition, 2005, vol. 6, p. 144.

30. Fu H , Pan C, Yao W and Zhu Y, J. Phys. Chem. B, 2005, 109, 22432.16853922

31. Rodriguez JA and Maiti A, J. Phys. Chem. B, 2000, 104, 3630.

32. Potrzebowski MJ , Blaszczyk J , Wieczorek MW and Klinowski J , J. Chem. Phys, 1999, 101, 8077.

33. Bredow T, Apra E, Catti M, Pacchioni G and Cluster G, Surf. Sci, 1998, 418, 150.

34. Casarin M , Maccato C and Vittadini A, Surf. Sci, 1997, 377-379, 587.

35. C. A. Scamehorn, N. M. Harrison and M. I. McCarthy, J. Chem. Phys, 1994, 101, 1547.

36. Zhang F, Yediler A and Liang X, Chemosphere, 2007, 67, 712.17188325

37. Bae E and Choi W, Environ. Sci. Technol, 2003, 37, 147.12542303

38. He JJ , Hagfeldt A and Lindquist SE , Langmuir, 2001, 17, 2743. 


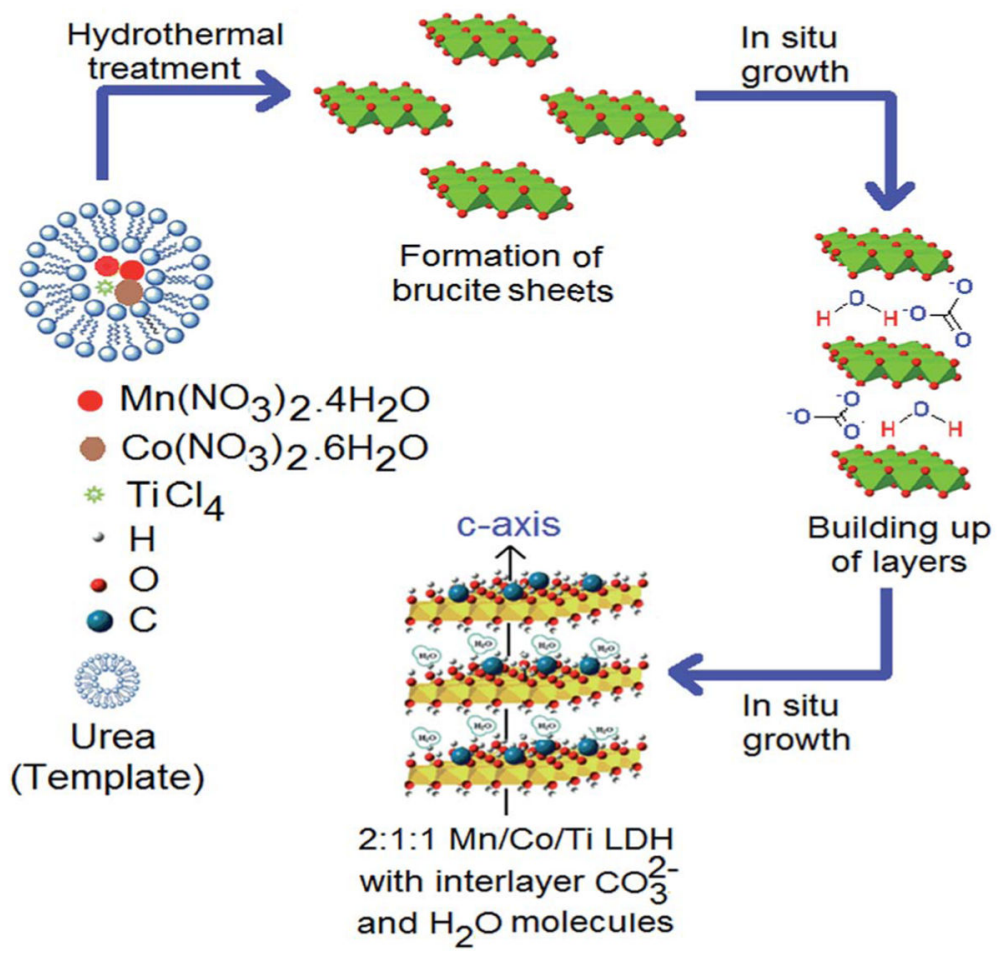

Fig. 1.

Scheme of hydrothermal synthesis of $2: 1: 1 \mathrm{Mn} / \mathrm{Co} / \mathrm{Ti} \mathrm{LDH}$. 


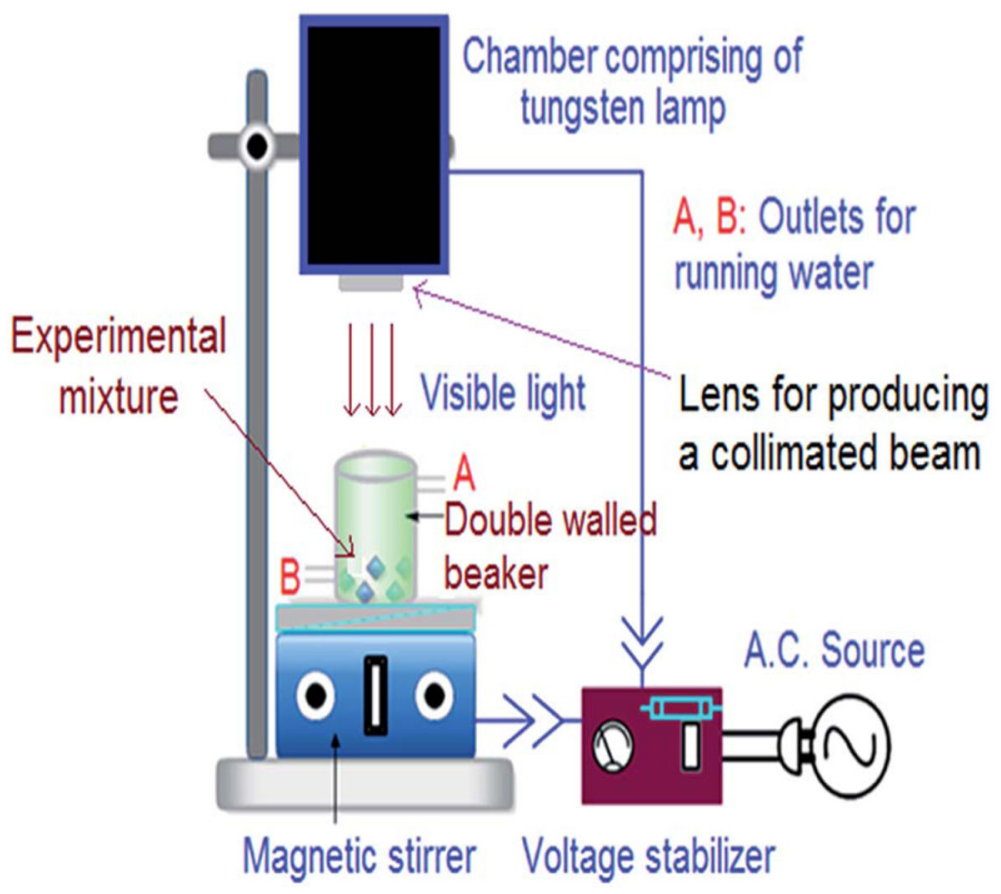

Fig. 2.

Schematic design of the core portion visible light photocatalytic device. 


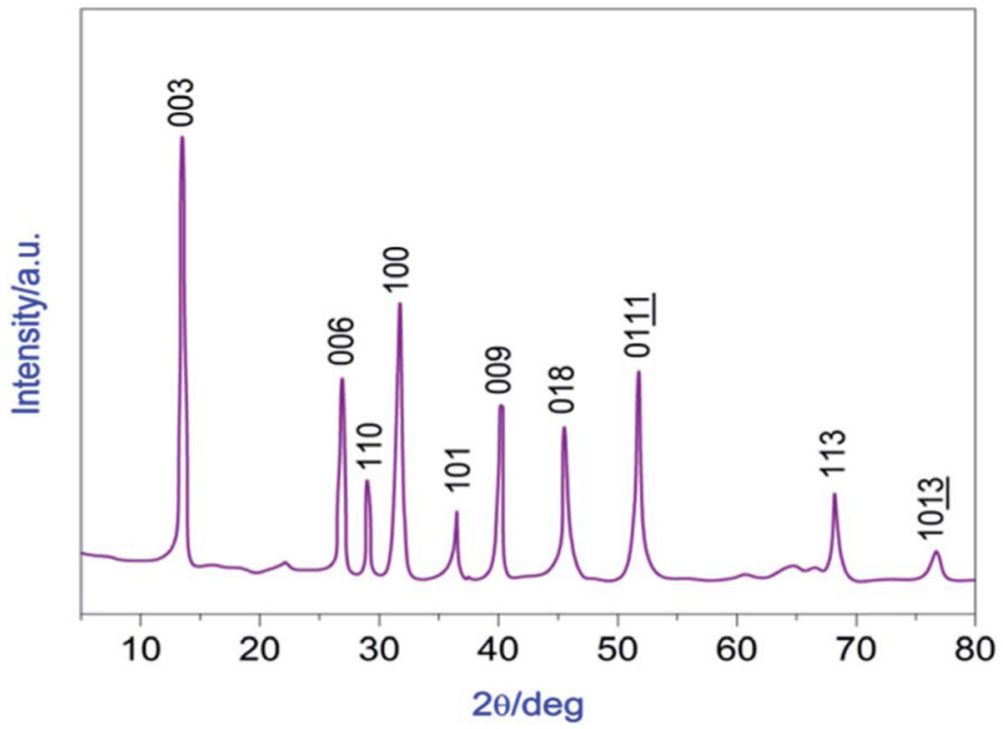

Fig. 3.

X-ray diffractogram of $2: 1: 1 \mathrm{Mn} / \mathrm{Co} / \mathrm{Ti} \mathrm{LDH}$. 

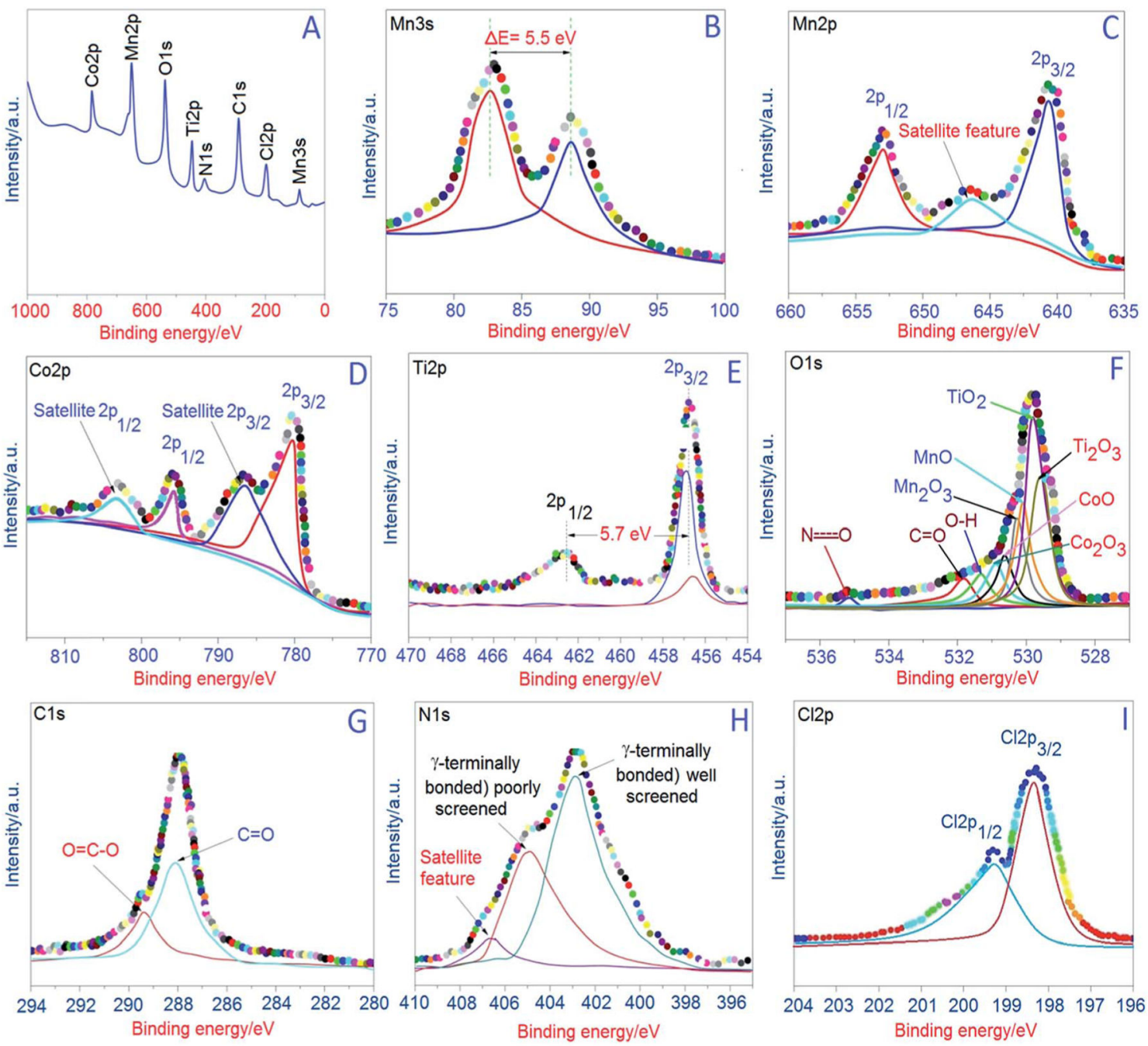

Fig. 4.

(A) Full scan XPS survey, (B) Mn 3s XPS line, (C) Mn 2p XPS line, (D) Co 2p XPS line, (E) Ti 2p XPS line, (F) O 1s XPS line, (G) C 1s XPS line, (H) N 1s XPS line, (I) Cl 2p XPS line of $2: 1: 1 \mathrm{Mn} / \mathrm{Co} / \mathrm{Ti} \mathrm{LDH}$. High resolution core level XPS lines are represented by color maps and the deconvoluted peaks are represented by solid lines. Deconvolution was performed with CASA XPS software (version 2.3.15). 

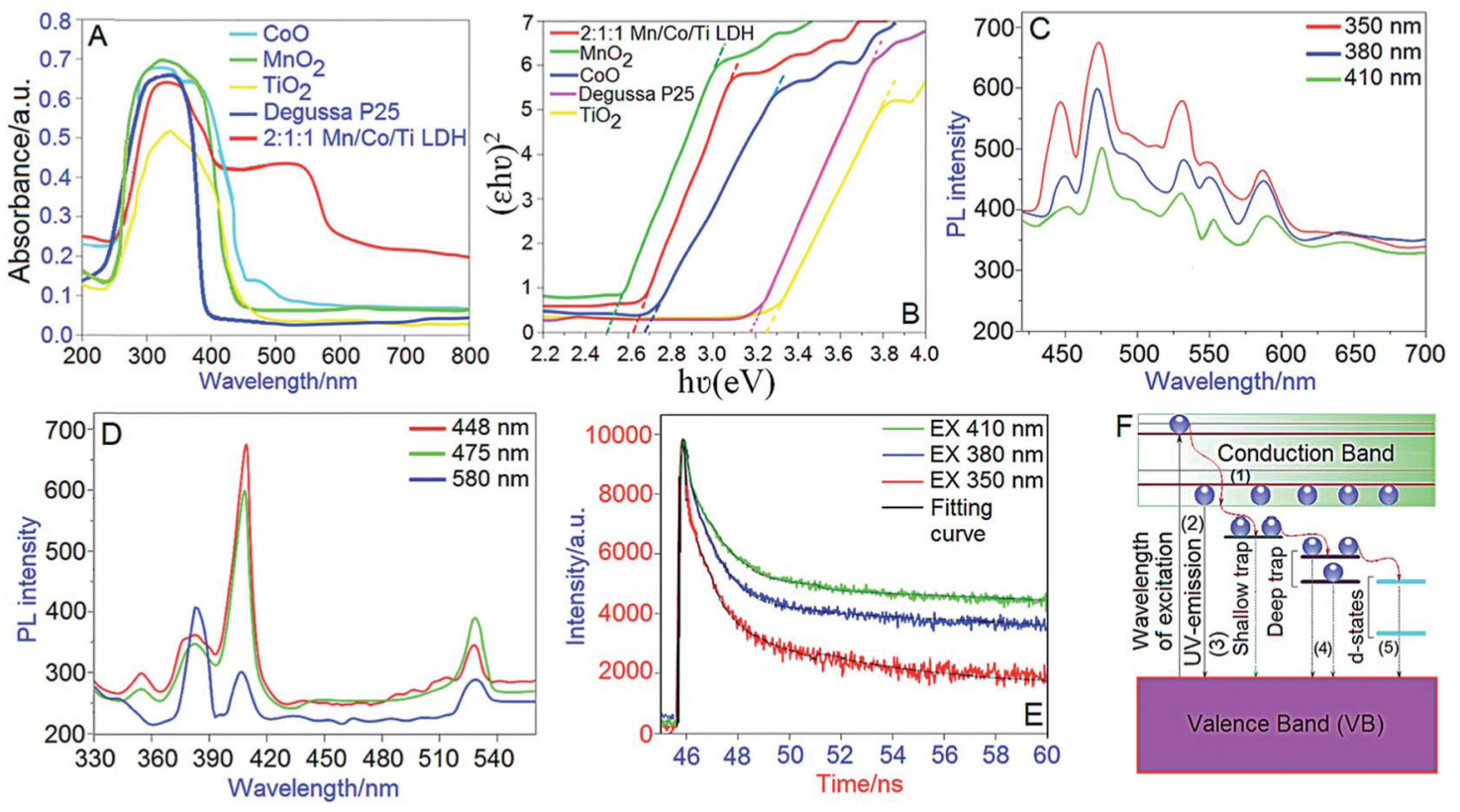

Fig. 5.

(A). UV-visible DRS (B) Tauc plots for calculation of band gap for Mn/Co/Ti LDH with commercial catalysts (C) photoluminescence emission spectra at different excitation wavelengths (D) photoluminescence excitation spectra at different emission wavelengths (E) time resolved emission spectra at different wavelengths (F) mechanism of photoluminescence emission of $2: 1: 1 \mathrm{Mn} / \mathrm{Co} / \mathrm{Ti} \mathrm{LDH}$. 

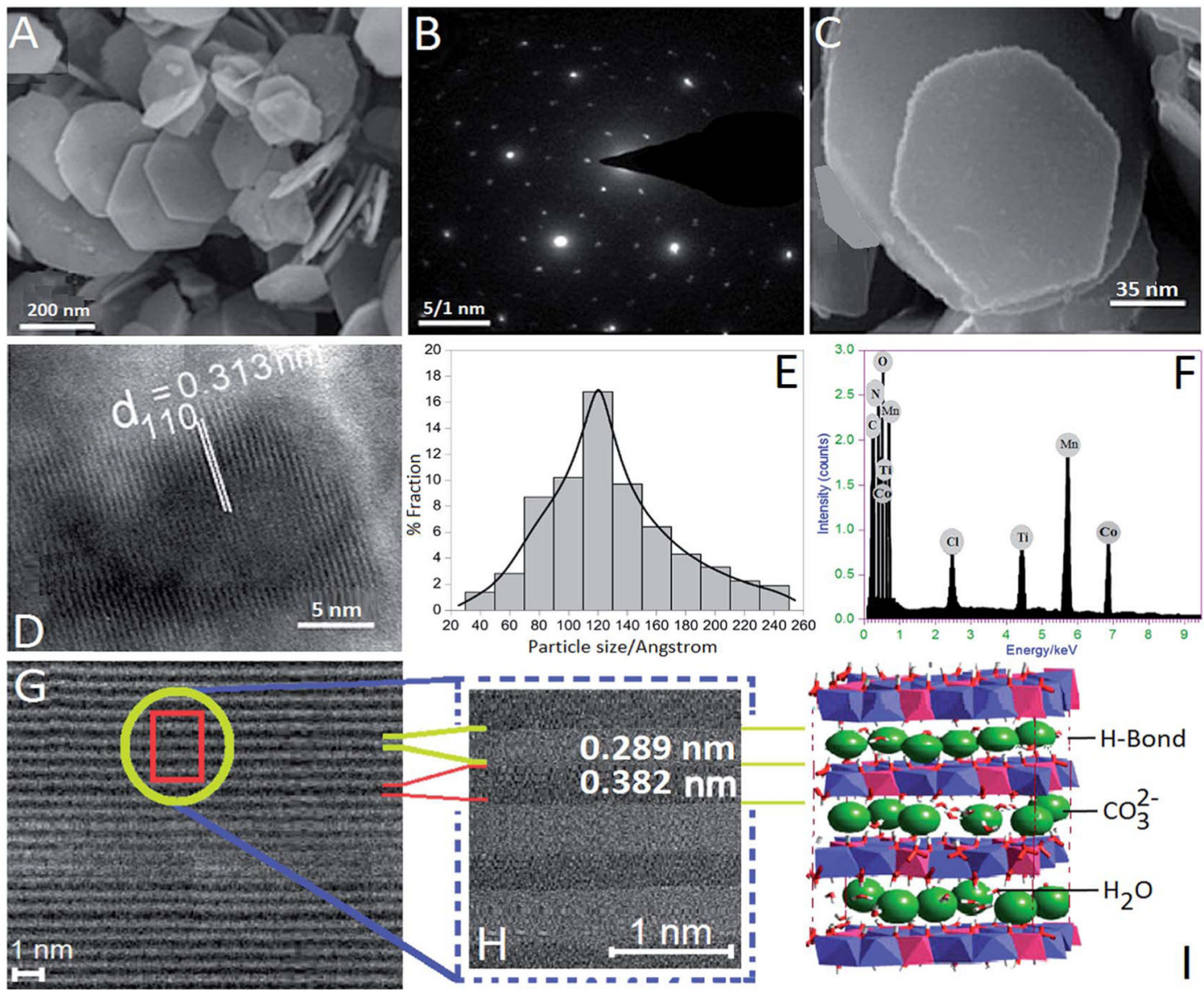

Fig. 6.

(A) TEM image (B) SAED pattern (C) high resolution TEM image (D) lattice fringe separation exhibited by TEM (E) histogram plot showing particle size distribution (F) EDS spectrum $(\mathrm{G}, \mathrm{H})$ high resolution cross sectional TEM images and its co-relation with (I) idealized LDH structure 

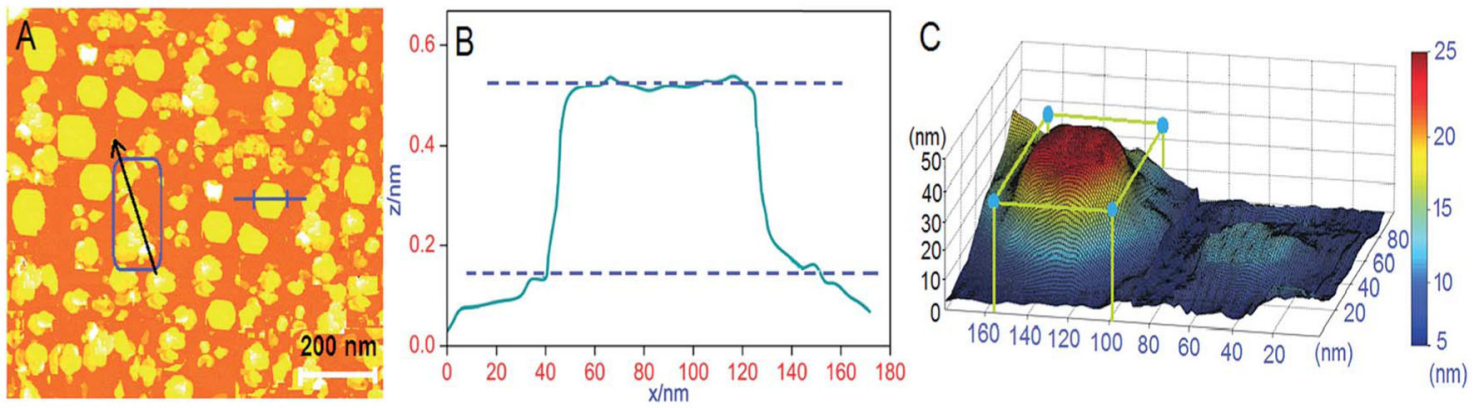

Fig. 7.

(A) AFM image (B), AFM height profile of a single brucite sheet, (C) 3D AFM total height profile of $\mathrm{Mn} / \mathrm{Co} / \mathrm{Ti} \mathrm{LDH}$. 

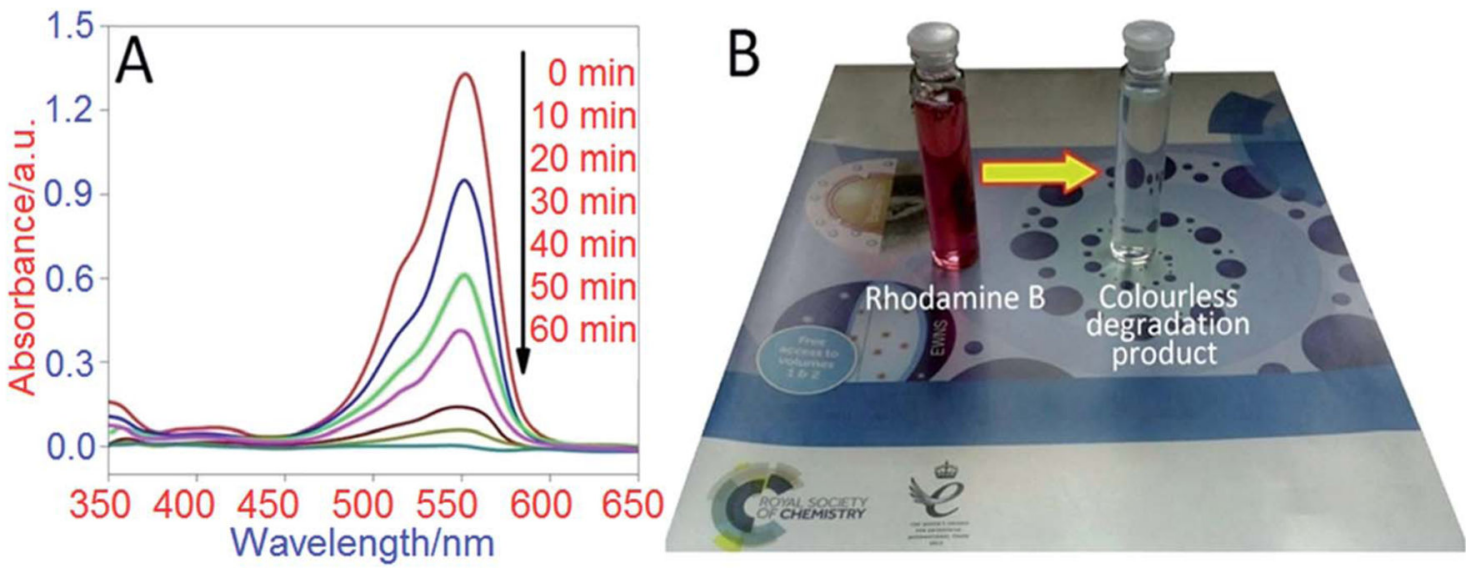

Fig. 8.

(A) UV-visible absorption spectra of Rhodamine B degradation by $\mathrm{Mn} / \mathrm{Co} / \mathrm{Ti} \mathrm{LDH}$ at different time intervals, (B) snapshot showing aqueous Rhodamine B and the colourless end products obtained after degradation by $\mathrm{Mn} / \mathrm{Co} / \mathrm{Ti} \mathrm{LDH}$. 

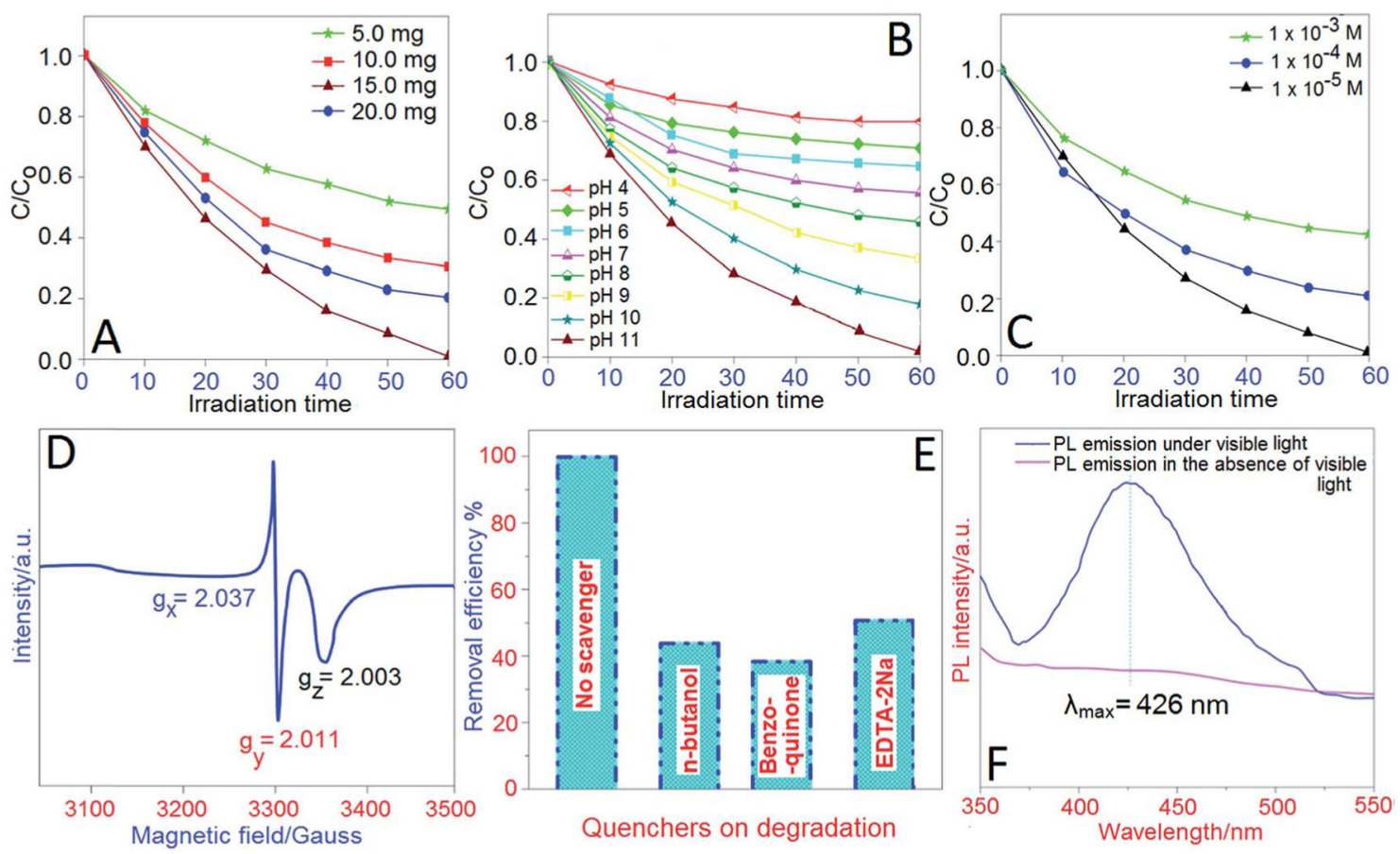

Fig. 9.

The influence of (A) LDH amount, (B) $\mathrm{pH}$, (C) initial dye concentration on photodegradation of aqueous $\mathrm{RhB}$. (D) EPR spectrum of the $\mathrm{LDH}$ for detection of $\left(\mathrm{O}_{2}{ }^{--}\right)$ species during photocatalysis, (E) roles of different active species on photodegradation of aqueous RhB, (F) photo-luminescence spectral changes with visible light irradiation over $\mathrm{Mn} / \mathrm{Co} / \mathrm{Ti} \mathrm{LDH}$ in the basic solution of terephthalic acid (TA) for detection of ${ }^{\bullet} \mathrm{OH}$ radicals. 


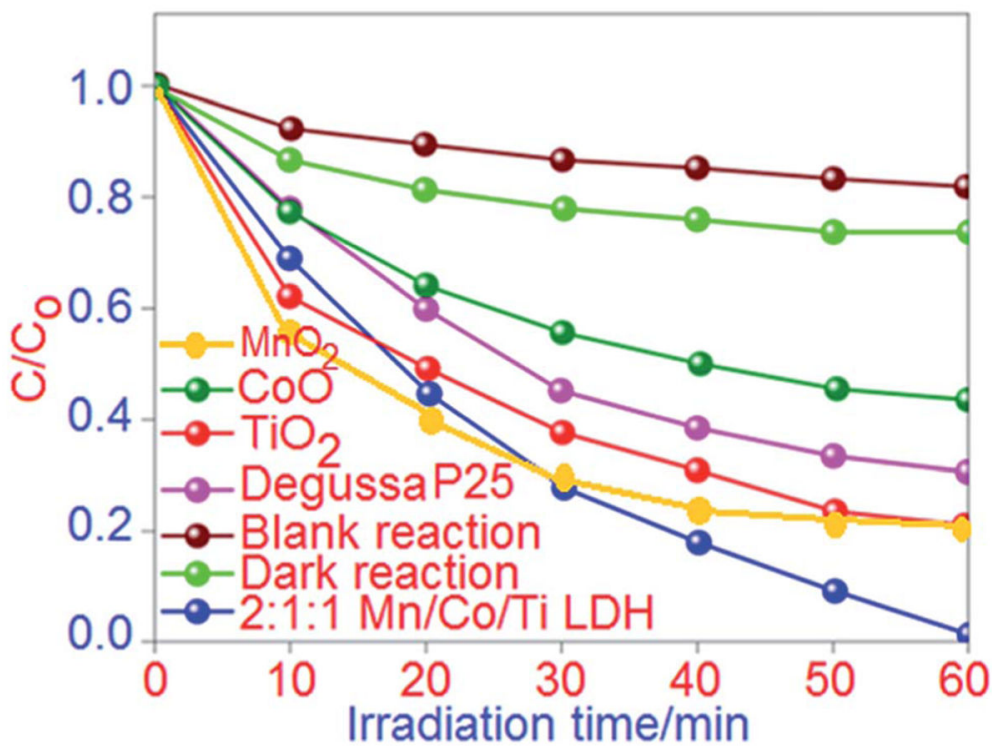

Fig. 10.

Comparative evaluation of $\mathrm{Mn} / \mathrm{Co} / \mathrm{Ti} \mathrm{LDH}$ and commercial catalysts for photodegradation of Rhodamine B by (under visible light and controlled conditions) over commercial catalysts. 


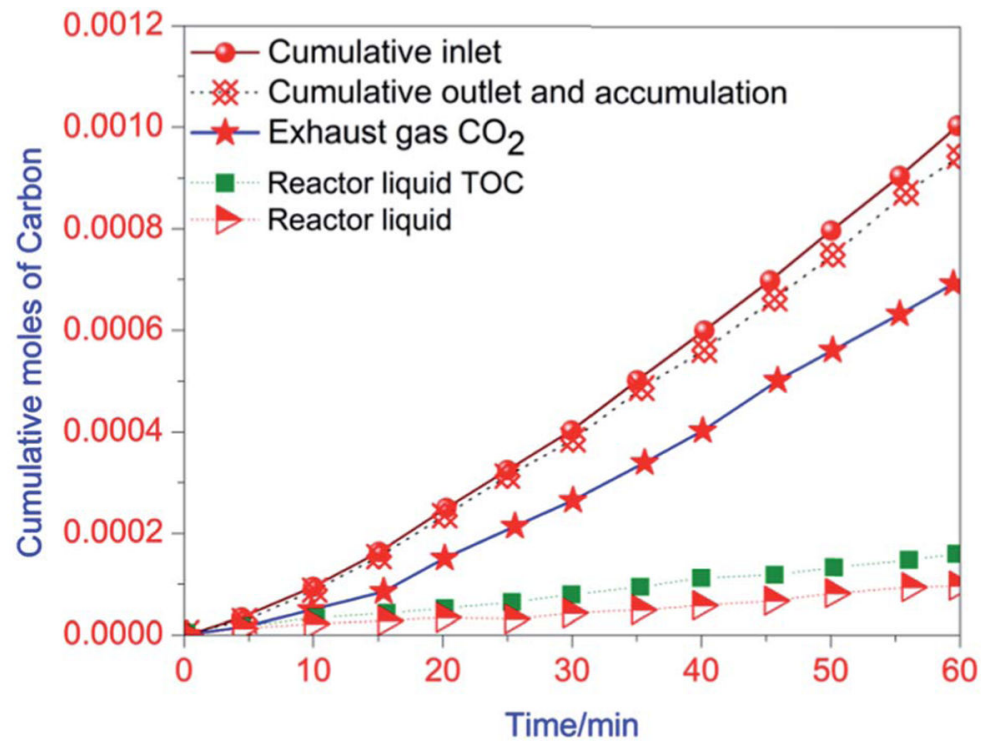

Fig. 11.

Cumulative moles of carbon in separate forms and media. 


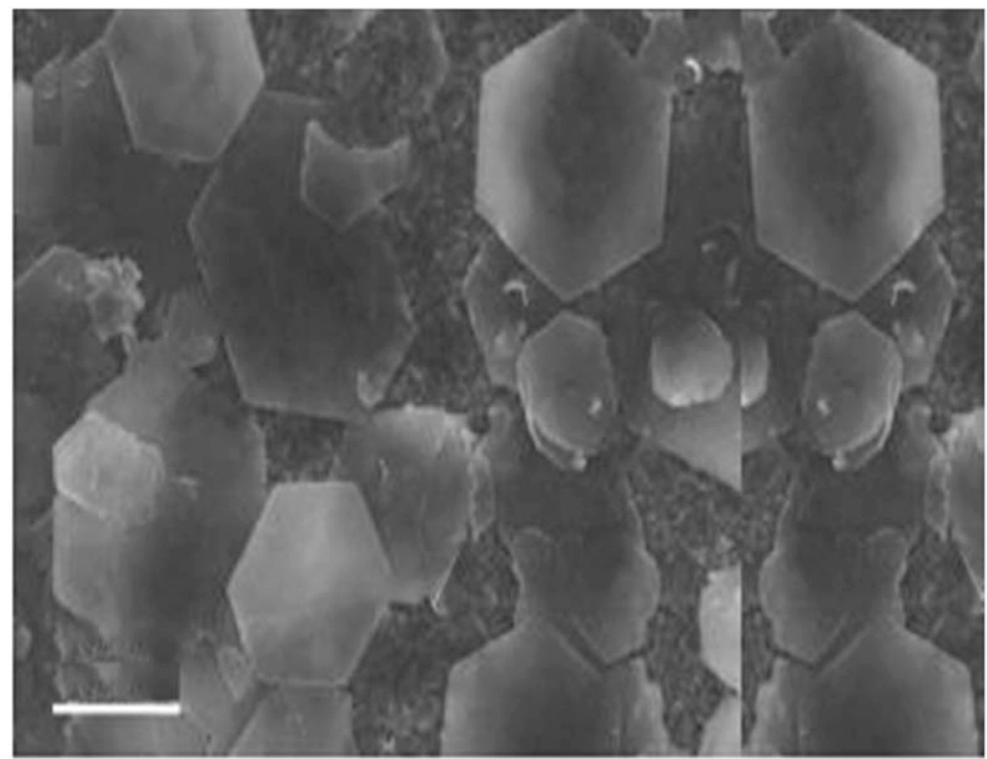

Fig. 12.

TEM image of the recovered LDH after the fifth photo-degradation cycle. 


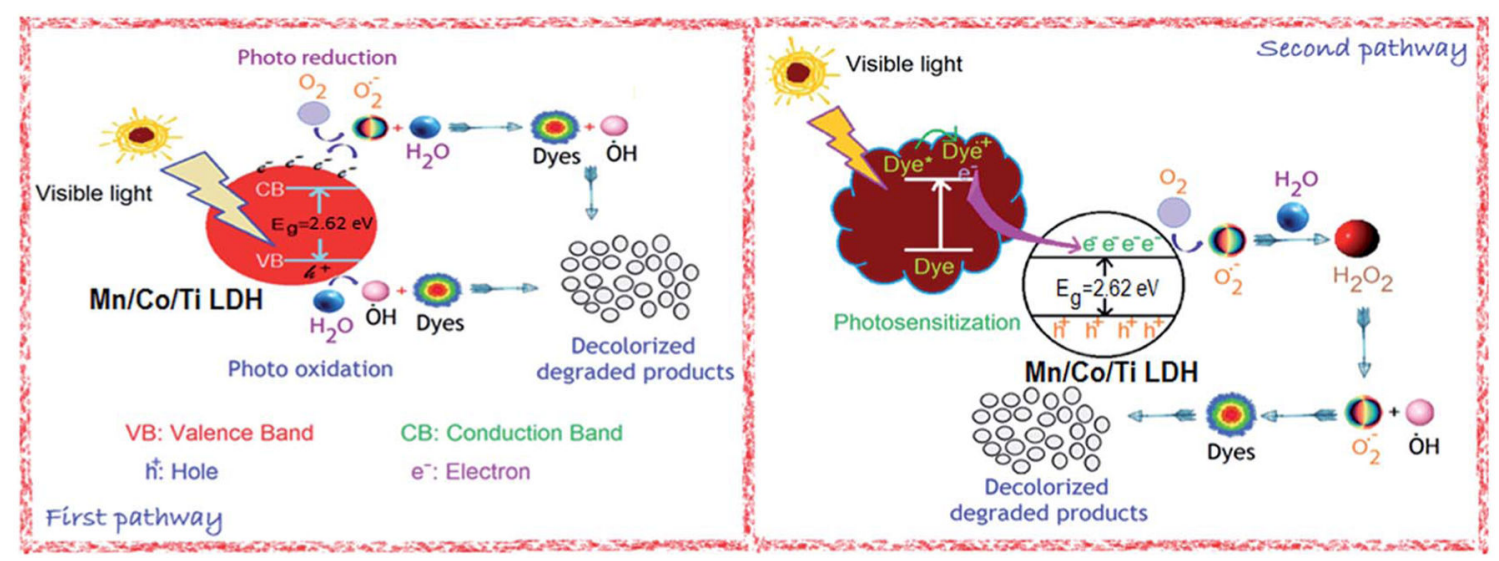

Fig. 13.

Schematic diagram showing the mechanistic pathways of $\mathrm{RhB}$ degradation by $\mathrm{Mn} / \mathrm{Co} / \mathrm{Ti}$ LDH under visible light irradiation. 


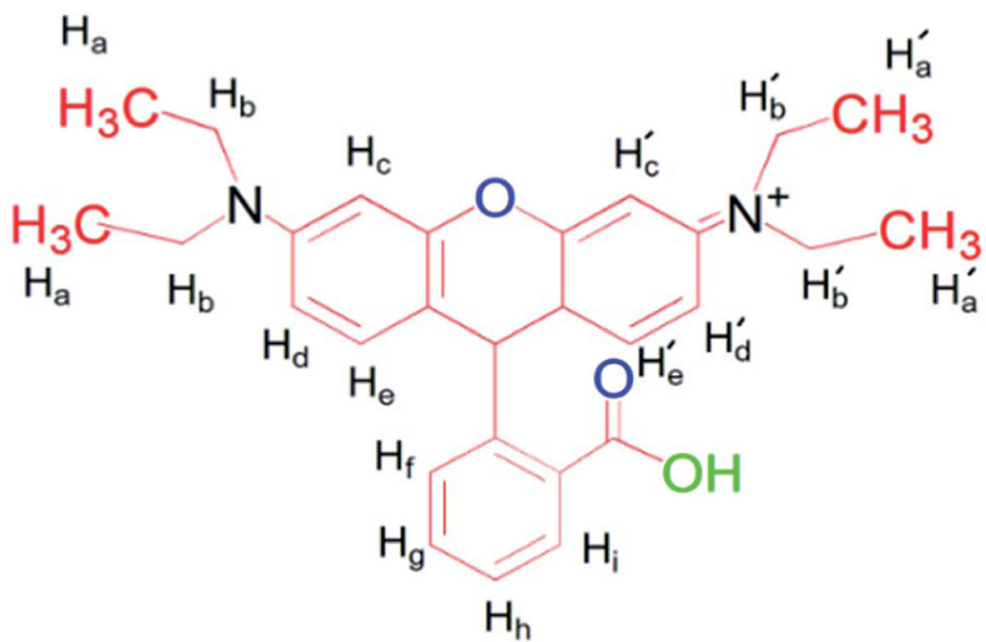

Fig. 14.

Structure of Rhodamine B showing different protons at different chemical environments. 


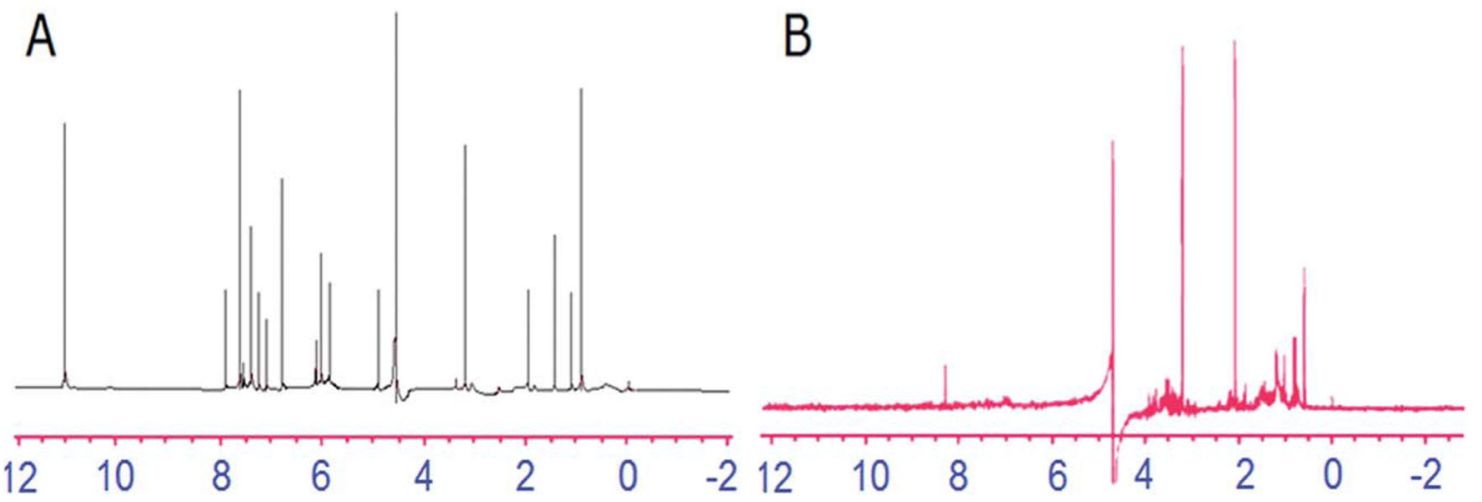

Fig. 15.

(A) ${ }^{1} \mathrm{H}-\mathrm{NMR}$ spectra of pure Rhodamine B. (B) ${ }^{1} \mathrm{H}-\mathrm{NMR}$ spectra of decolorized end products, obtained after removing $\mathrm{Mn} / \mathrm{Co} / \mathrm{Ti} \mathrm{LDH}$ using $60 \mathrm{~min}$ of visible light irradiation. 


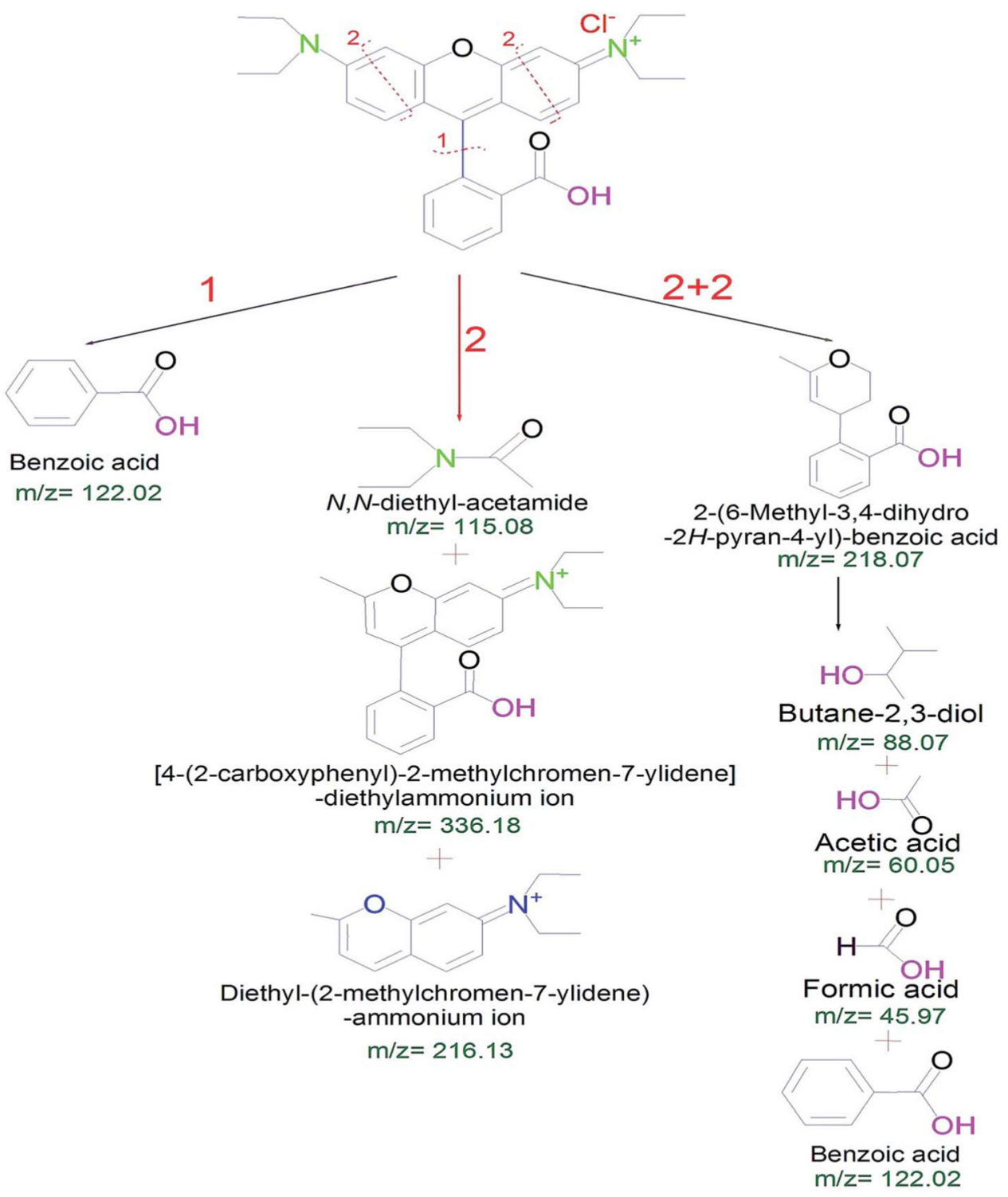

Fig. 16.

Photodegradation pathway of Rhodamine B (RhB) catalyzed by $2: 1: 1 \mathrm{Mn} / \mathrm{Co} / \mathrm{Ti} \mathrm{LDH}$ under visible light irradiation. 
Table 1

Lifetime of photo-generated charge carriers of $\mathrm{Mn} / \mathrm{Co} / \mathrm{Ti} \mathrm{LDH}$ at different wavelengths

\begin{tabular}{lllllll}
\hline $\boldsymbol{\lambda}_{\text {ex }}(\mathbf{n m})$ & $\boldsymbol{A 1}$ & $\boldsymbol{\tau}_{\mathbf{1}}(\mathbf{n s})$ & $\boldsymbol{A}_{\mathbf{2}}$ & $\boldsymbol{\tau}_{\mathbf{2}}(\mathbf{n s})$ & $\boldsymbol{\tau}(\mathbf{n s})$ & $\mathrm{X}^{\mathbf{2}}$ \\
\hline 370 & 7271.3 & 6.219 & 3042.3 & 4.043 & 2.74 & 0.97 \\
390 & 5582.4 & 2.258 & 4378.1 & 5.718 & 4.58 & 0.98 \\
410 & 6023.6 & 6.314 & 4696.7 & 4.157 & 5.68 & 0.96
\end{tabular}


Table 2

EDX elemental composition of $2: 1: 1 \mathrm{Mn} / \mathrm{Co} / \mathrm{Ti} \mathrm{LDH}$

\begin{tabular}{llll}
\hline Elements & Weight\% & Atomic\% & Mn/Co/Ti (ratio) \\
\hline C K & 1.82 & 1.28 & $2.06: 1.04: 1$ \\
N K & 2.50 & 1.84 & \\
O K & 9.64 & 2.58 & \\
Cl K & 3.85 & 2.15 & \\
Mn K & 41.35 & 46.34 & \\
Co K & 20.78 & 23.31 & \\
Ti K & 20.06 & 22.50 & \\
Total & $\mathbf{1 0 0}$ & $\mathbf{1 0 0}$ & \\
& & &
\end{tabular}


Table 3

Efficiency of degradation of aqueous RhB by LDH and commercial catalysts (under visible light and controlled conditions)

\begin{tabular}{ll}
\hline Catalyst/conditions & Degradation efficiency $(\%)$ \\
\hline 2:1:1 Mn/Co/Ti LDH & 99.8 \\
$\mathrm{TiO}_{2}$ & 78 \\
$\mathrm{MnO}_{2}$ & 75 \\
$\mathrm{CoO}$ & 50 \\
Degussa P25 & 84 \\
Blank reaction & 19 \\
Dark reaction & 25
\end{tabular}


Table 4

EDX elemental composition of the spent $\mathrm{Mn} / \mathrm{Co} / \mathrm{Ti} \mathrm{LDH}$ after the fifth cycle of photodegradation

\begin{tabular}{llll}
\hline Elements & Weight $\%$ & Atomic\% & Mn/Co/Ti (ratio) \\
\hline C K & 1.98 & 1.11 & $2.06: 1.04: 1$ \\
N K & 2.95 & 1.35 & \\
O K & 9.58 & 2.38 & \\
Cl K & 3.85 & 2.87 & \\
Mn K & 41.48 & 46.85 & \\
Co K & 20.09 & 22.75 & \\
Ti K & 20.07 & 22.69 & \\
Total & $\mathbf{1 0 0}$ & $\mathbf{1 0 0}$ &
\end{tabular}

